\title{
Localized coherence of freak waves
}

\author{
Arnida L. Latifah ${ }^{1,2}$ and E. van Groesen ${ }^{1,3}$ \\ ${ }^{1}$ University of Twente, Enschede, the Netherlands \\ ${ }^{2}$ Indonesian Institute of Sciences, Bandung, Indonesia \\ ${ }^{3}$ LabMath-Indonesia, Bandung, Indonesia \\ Correspondence to: Arnida L. Latifah (a.1.latifah@utwente.nl)
}

Received: 13 May 2016 - Published in Nonlin. Processes Geophys. Discuss.: 17 May 2016

Revised: 18 July 2016 - Accepted: 1 August 2016 - Published: 16 September 2016

\begin{abstract}
This paper investigates in detail a possible mechanism of energy convergence leading to freak waves. We give examples of a freak wave as a (weak) pseudo-maximal wave to illustrate the importance of phase coherence. Given a time signal at a certain position, we identify parts of the time signal with successive high amplitudes, so-called group events, that may lead to a freak wave using wavelet transform analysis. The local coherence of the critical group event is measured by its time spreading of the most energetic waves. Four types of signals have been investigated: dispersive focusing, normal sea condition, thunderstorm condition and an experimental irregular wave. In all cases presented in this paper, it is shown that a high correlation exists between the local coherence and the appearance of a freak wave. This makes it plausible that freak waves can be developed by local interactions of waves in a wave group and that the effect of waves that are not in the immediate vicinity is minimal. This indicates that a local coherence mechanism within a wave group can be one mechanism that leads to the appearance of a freak wave.
\end{abstract}

\section{Introduction}

Understanding the mechanism of the freak wave phenomenon is intriguing for scientists, engineers and mariners. The mechanisms that lead to freak waves are understandably diverse and it is not surprising that different freak waves exhibit different qualitative features (Liu and Mori, 2000). A review of the existing mechanisms of freak waves was presented by Pelinovsky and Kharif (2008) and Slunyaev et al. (2011).
We consider freak waves in unidirectional wave fields which satisfy the common definition of a freak wave, namely that the wave height exceeds approximately 2 times the significant wave height $\left(H_{\mathrm{s}}\right)$ or that the crest height exceeds $1.25 H_{\mathrm{s}}$ (Kharif and Pelinovsky, 2006, 2003; Olagnon and van Iseghem, 2000; Dysthe et al., 2008). Freak waves that are dominantly generated from wave energy convergence as a consequence of the random superposition of many wave components with not necessarily strong nonlinearity is still under discussion (Wang et al., 2015; Onorato et al., 2013; Garret and Gemmrich, 2009; Gemmrich and Garrett, 2008; Slunyaev et al., 2005; Muller et al., 2005). Different from some papers (Haver, 2004; Kharif and Pelinovsky, 2003; Pelinovsky et al., 2000), in which a freak wave is discussed as an accidental event from nowhere that appears and disappears suddenly, we discuss freak waves in (mainly) random wave fields that exhibit long-life gradual growth and decay. Latifah and van Groesen (2012) described and predicted freak waves by measuring the degree of phase coherence from a given time series at one position. It is the phase variance over an interval of the dominant wave frequencies. In this paper, we investigate the local coherence computed from the local time spreading of the most energetic waves, which is determined by wavelet transform. Nowadays, wavelet transformation is widely applied to analyze freak waves (Hu et al., 2015; Kwon et al., 2015; Cherneva and Guedes Soares, 2014; Bai et al., 2015; Wang et al., 2015; Wu et al., 2010), as it has wider applicability than Fourier techniques (Lin and Liu, 2004).

In the study of Slunyaev et al. (2005), the calculation of the first derivative of the local group velocity in the time series shows the presence of regions of strong wave convergence or divergence near freak events where strong modulations 
occurs. However, the question about the origin of the freak wave, whether it is naturally contained in the wave trains or induced by Benjamin Feir instability, is still open. Pelinovsky et al. (2011) discussed a freak wave of the solitary-like shape that is originated from the wave packet and is based on the dispersive focusing of unidirectional wave packets. In addition to the references cited above, we will contribute in understanding the process and the origin of freak wave appearance in random wave fields that is mainly based on dispersive effects. In realistic sea states, a directional spreading could possibly influence dispersive focusing effects (Prevosto, 1998). Also Johannessen and Swan (1999) concluded that the introduction of directionality significantly reduces the nonlinearity of wave groups. That nonlinearity gives little or no extra amplitude compared to linear extreme events, but the changing shape of the extreme crest was also observed by Adcock et al. (2015). In this paper, we will not take directional spreading into account, but will restrict to long-crested, unidirectional waves.

In unidirectional linear waves, the focusing due to dispersion is one mechanism that causes a freak wave (Porubov et al., 2005; Slunyaev et al., 2005; Kharif et al., 2001; Brown and Jensen, 2001; Pelinovsky et al., 2000; Baldock et al., 1996). If short waves with small group velocities are initially located in front of long waves having large group velocities, the long waves will overtake the short waves with increasing time and large-amplitude waves can appear. Afterwards, the long waves will be in front of the short waves and the amplitude of the wave train will decrease (Kharif and Pelinovsky, 2003). This mechanism is observed in the type of dispersive focusing waves which are often used in hydrodynamic laboratories (Merkoune et al., 2013; Brown and Jensen, 2001; Clauss, 2002; Shemer et al., 2007, 2005; Grue et al., 2003). In random waves, this mechanism could also trigger a freak wave, but it is not as clear as in the dispersive focusing case. In the study of Wang et al. (2015), they presented a freak wave in a random wave field that was generated from two successive wave groups with different main frequencies and the higher frequency waves are in front of the others.

According to the study of Sergeeva et al. (2014) and Sergeeva and Slunyaev (2013), most of the long-living freak waves often occur on the background of intense wave groups. The evolution of modulated wave groups over large spatial and temporal scales were also a concern in the study of Viotti et al. (2013) and Grimshaw et al. (2001). Recently Cousins and Sapsis $(2014,2016)$ and Ruban (2013) underlined that the appearance of extreme events can be triggered by focusing energy in localized wave groups. Therefore, to identify the group profiles that can be the origin of freak waves appearance, they used envelope equations and identified the envelope of the dominant groups associated with the length scale and amplitude by a group detection algorithm. Further, they computed the probability of the group to develop an extreme event. The evolution of the freak waves is summarized into focusing-defocusing process of energy. During the generation, a single wave absorbs energy from neighboring waves, increases its amplitude, reaches a maximum and then returns its energy back to other waves (Xia et al., 2015; Slunyaev et al., 2005). According to Kharif et al. (2009), the transient change of the local energy of wave groups can be caught by wavelet analysis better than Fourier analysis.

In this paper, we will consider the appearance of freak waves in evolving wave groups in space and time. The waves are generated from a signalling problem: at the influx position, say $x_{0}$, a given time signal $\eta\left(x_{0}, t\right)$ is forced in one direction, the positive $x$ axis. The resulting waves $\eta(x, t)$ may show a freak wave at certain time and space $\left(x_{f}, t_{f}\right)$ at which the amplitude is larger than $1.25 H_{\mathrm{s}}$, which is taken as the definition of a freak wave in the rest of this paper. We will investigate this appearance by concentrating on successive high amplitudes in the initial signal, which will be called critical group events. We will apply the wavelet analysis for the identification of the energy spectral distribution in the group events.

This paper is organized into five sections starting with this introduction. Section 2 starts with a motivation to investigate the local coherence by showing the rapid decrease of the maximal amplitude when the coherence is decreased. Wavelet transformation is then described and shown to be better capable than Fourier methods to analyze the local phase of a wave. Section 3 starts with the selection of possible freak waves by estimating the critical group events from the influx signal that can lead to freak waves further downstream. The propagation of the most energetic group is then simulated to show the successive local energy convergence. We introduce quantitative measures of local coherence as one tool to predict the freak wave appearance. Using numerical simulations of linear and nonlinear waves with the $\mathrm{AB}$ equation described in Appendix A (van Groesen and Andonowati, 2007; van Groesen et al., 2010), we compute the wave evolution and measure the local coherence of the time signal at several positions. We consider various wave types, a dispersive focusing wave and irregular waves, synthetic and experimental signals from the MARIN hydrodynamic laboratory in Sect. 4. Conclusions are formulated in the final section.

\section{Coherence and wavelet transform}

In this section, we will start to motivate and illustrate the role of coherence by considering maximal, pseudo-maximal (pm) and weak pseudo-maximal (wpm) signals that can describe freak waves. In Latifah and van Groesen (2012), the notion of a pseudo-maximal signal was introduced for which the phases of all frequencies were band limited. Below, we also consider a less restrictive notion of weak pseudo-maximal signal, by restricting the phase only for the most energycarrying modes. The measure of phase coherence in these concepts uses Fourier transform that represents the energy and the phase as function of the frequency. In Sect. 2.2, we 
describe the wavelet transform that is used in this paper to extract the local energy spectral distribution and the local phase as the time-frequency information of a given signal. Plots of the energy distribution over the frequencies will show that the wavelet transform improves results obtained with Fourier transform.

\subsection{Signal coherence}

Waves in the ocean at a specific position are described by a time signal. An irregular signal will have phases that are commonly understood to be uniformly distributed in $(-\pi, \pi]$. Previous study (Latifah and van Groesen, 2012) defined maximal waves and pseudo-maximal waves. A maximal wave is a wave with all phases zero and has maximal amplitude equal to the integration of its two-sided absolute spectrum. Thus, we call a signal with all phases zero at some time ( say $t=0$ ) a maximal signal, as

$\operatorname{MS}(t)=\int\left|\eta_{0}(\omega)\right| \cos (\omega t) \mathrm{d} \omega$

At $t=0$, all wave components contribute to a constructive interference, hence

$\operatorname{MS}(0)=\int\left|\eta_{0}(\omega)\right| \mathrm{d} \omega$

This is the highest amplitude that is possible for given spectrum, $\eta_{0}(\omega)$. In view of the assumption of uniform distribution of the phases, the chance for such a maximal wave vanishes.

A pseudo-maximal (pm) wave is a partly coherent wave, that is in between a completely irregular wave and a fully coherent maximal wave. For a given signal with random phase $\theta(\omega) \in(-\pi, \pi]$ as a function of wave frequencies with $\theta(\omega)=-\theta(-\omega)$, we consider a pm signal as the signal for which the phases are restricted for certain $\alpha \in(0,1)$ to the phases $\theta_{\alpha}(\omega)=\alpha \theta(\omega)$, as

$\left[\eta_{\mathrm{pm}}(t)\right]_{\alpha}=\int\left|\eta_{0}(\omega)\right| \cos \left(\theta_{\alpha}(\omega)-\omega t\right) \mathrm{d} \omega$

By taking a fraction $\alpha$ of the random phase, the maximal amplitude decreases and the background increases for increasing $\alpha$. For $\alpha=0$, it is a maximal wave with coherent phases while for $\alpha=1$ it is an irregular wave and the freak wave may disappear completely.

The phases of all frequencies in a pm signal are constrained as $|\theta(\omega)|<\alpha \pi$. We now define a weak pseudomaximal (wpm) signal, $\eta_{\mathrm{r}}(t)$, by restricting the phases of only the frequencies of large energy-carrying modes (see Fig. 1 for an illustration). We also illustrate the importance of such restrictions for coherence by plotting the maximal, pm and wpm signals of a given Jonswap spectrum in Fig. 2.

The restriction of wpm signal is typically for frequencies within one (or a half) standard deviation (SD) around the

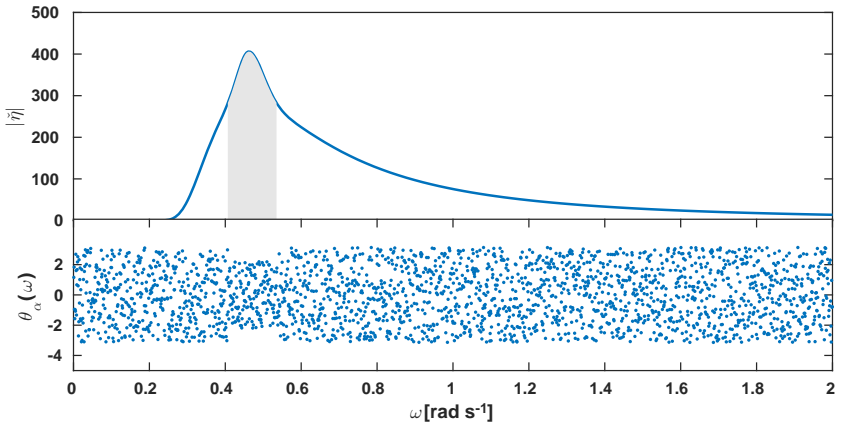

Figure 1. A Jonswap spectrum with restricted random phases, $\theta_{\alpha}(\omega)$. The shaded area represents the energy-carrying modes (restricted by a half standard deviation).

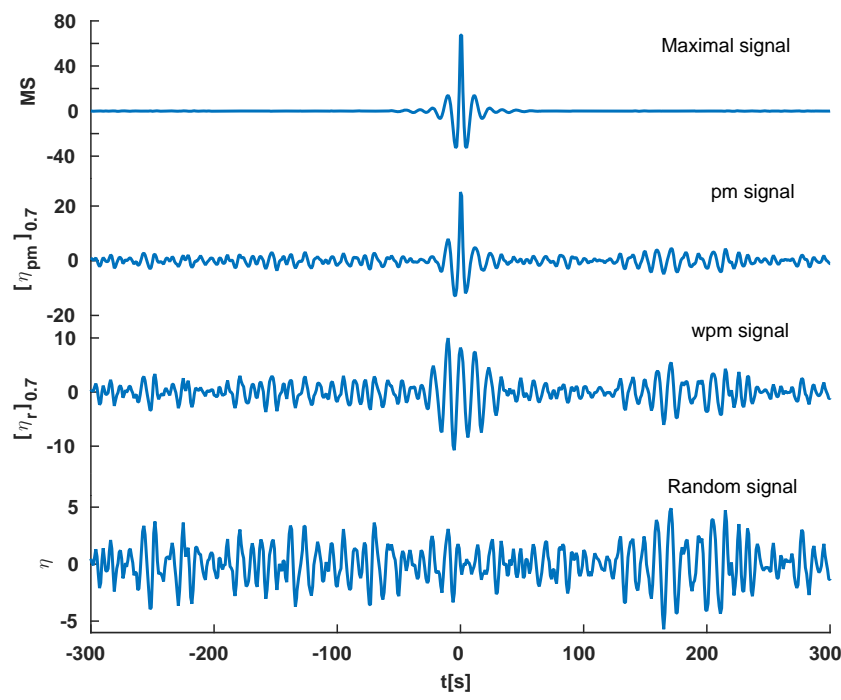

Figure 2. Shown are plots from up to down of a maximal, pseudomaximal and weak pseudo-maximal signal corresponding to the same random signal at the bottom. The random signal corresponds to a Jonswap spectrum with $H_{\mathrm{S}}=6.3 \mathrm{~m}$ and $\gamma=1.9$. The pm and wpm signals correspond to the value $\alpha=0.7$.

mean frequency, $\left|\omega-\omega_{\mathrm{m}}\right|<\sigma_{\omega}$ or less. Then we consider a signal for $\alpha=[0,1]$ and define $\theta_{\alpha}$ as

$\theta_{\alpha}(\omega)= \begin{cases}\alpha \theta(\omega), & \text { for }\left|\omega-\omega_{\mathrm{m}}\right|<\sigma_{\omega} \\ \theta(\omega), & \text { elsewhere }\end{cases}$

and get a signal that has maximal amplitude less than the maximal amplitude of the pm signal:

$\left[\eta_{\mathrm{r}}(t)\right]_{\alpha}=\int_{-\infty}^{\infty}|\eta(\omega)| \cos \left(\theta_{\alpha}(\omega)-\omega t\right) \mathrm{d} \omega \leq\left[\eta_{\mathrm{pm}}(0)\right]_{\alpha}$

In general, the mean frequency is not necessarily equal to the peak frequency because the spectrum of waves that is usually of Jonswap shape is not symmetric around the peak frequency. 


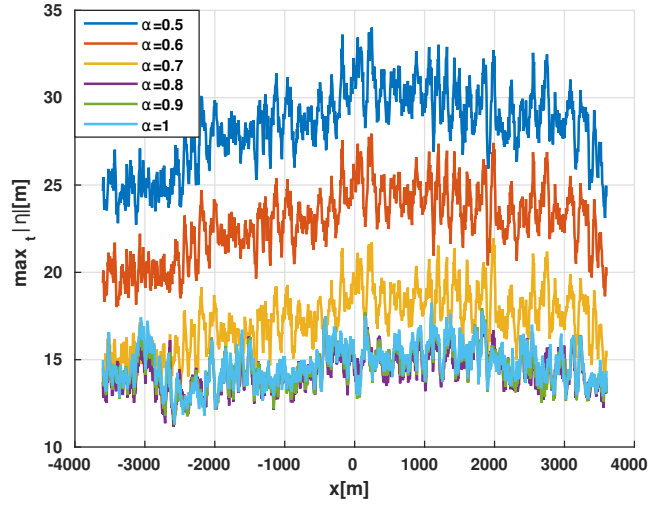

(a)

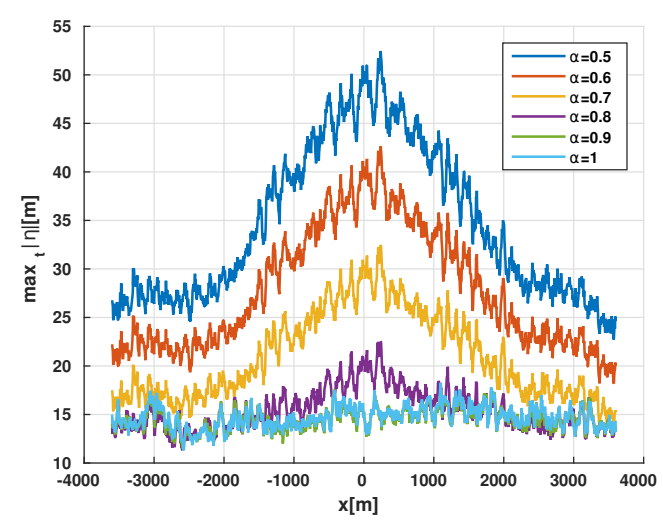

(b)

Figure 3. The maximal temporal amplitude of the linear evolution of the wpm signal for various values of $\alpha$. Panel (a) corresponds to restricting the phases to a quarter $\mathrm{SD},\left|\omega-\omega_{\mathrm{p}}\right|<0.25 \sigma_{\omega}$ and (b) for a half $\mathrm{SD},\left|\omega-\omega_{\mathrm{p}}\right|<0.5 \sigma_{\omega}$.

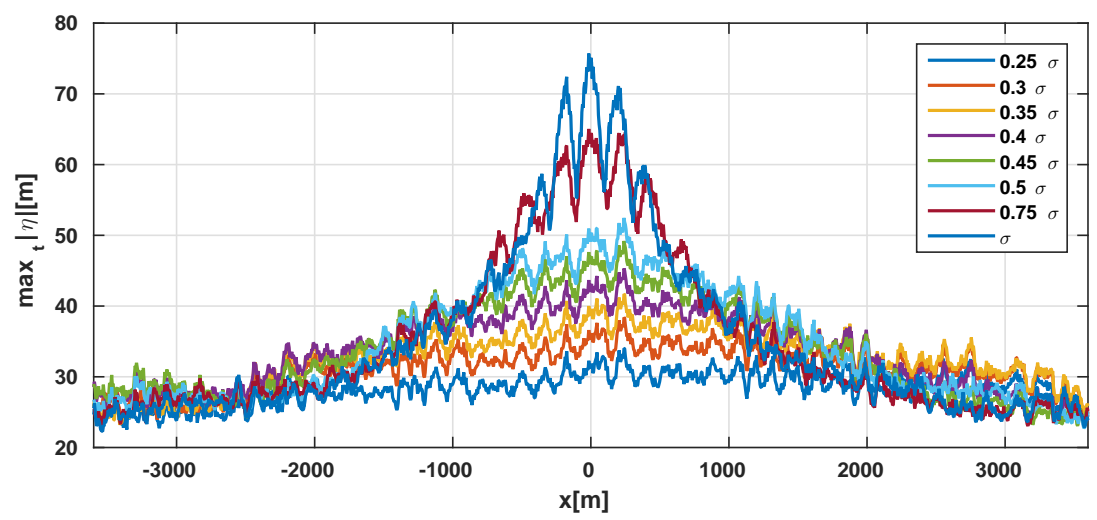

Figure 4. The maximal temporal amplitude of the linear evolution of the wpm signal with $\alpha=0.5$ for various fractions of the standard deviation $\sigma_{\omega}$.

Figure 3 illustrates that the value of $\alpha$ significantly affects the maximal crest height and the wave evolution along the $x$ axis. The smaller the value of $\alpha$, the higher the value of the generated crest. On the other hand, variations in $\sigma_{\omega}$ influence much less the maximum elevation of the influx signal. In any case, the wave evolution is tremendously affected and the maximum amplitude during the evolution can be much higher for larger $\sigma_{\omega}$. In Fig. 4 it is shown that at an influx position $(x \approx-3600 \mathrm{~m})$, the maximum amplitudes are quite the same for various fractions of SD, but near the focusing position a larger fraction of SD produces a higher maximum amplitude. This is the consequence of the fact that the larger fraction of SD gives more wave components with coherent phases.

Although the signal coherence can describe and measure the appearance of freak waves, the concepts use the whole interval of the time signal. However, not the whole interval will contribute in generating a freak wave since the waves propagate with their own group and phase velocity. The freak wave will be generated from local waves' interaction. Therefore, we will investigate the local energy propagation using wavelet transformation. This is expected to give a more refined measure of the appearance of the freak wave.

\subsection{Wavelet transform}

In Fourier analysis we transform a function that depends on time into a function that depends on the frequency as a single variable. Given a time signal $\eta(t)$, Fourier transformation gives the relations

$$
\begin{aligned}
\eta(\omega) & =\frac{1}{2 \pi} \int \eta(t) e^{i \omega t} \mathrm{~d} t \\
\eta(t) & =\int \eta(\omega) e^{-i \omega t} \mathrm{~d} \omega \\
& =2 \int_{0}^{\infty}|\eta(\omega)| \cos (\omega t+\theta(\omega)) \mathrm{d} \omega .
\end{aligned}
$$

The Fourier transform of $\eta(t)$ is the complex valued function $\eta(\omega)=|\eta(\omega)| e^{i \theta(\omega)}$, in which $|\eta(\omega)|$ is the amplitude spectrum and $\theta(\omega)$ is the phase of the signal. The spectral energy density of the signal is defined by $|\eta(\omega)|^{2}$ that describes how 

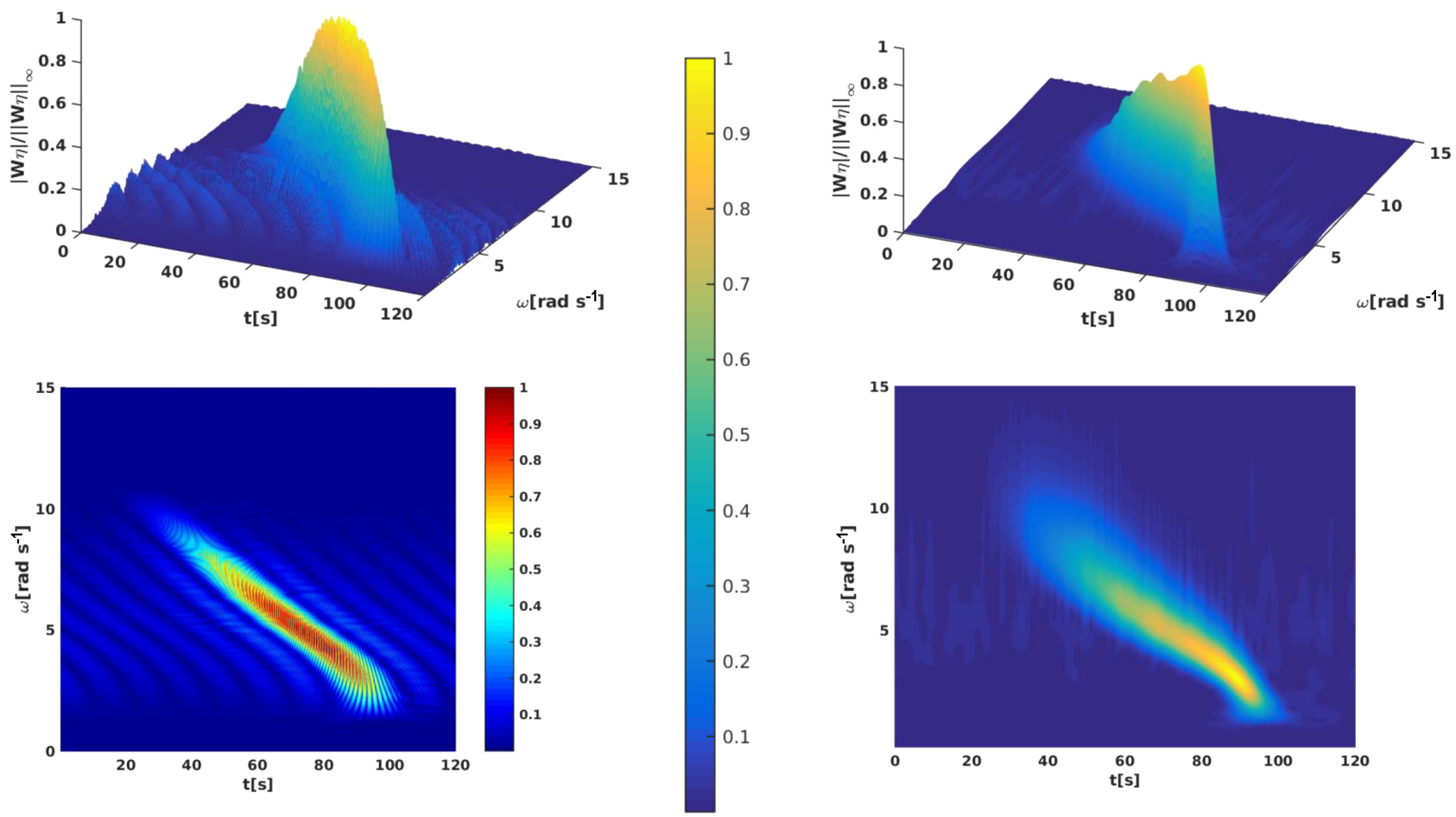

Figure 5. Shown is the distribution of the local energy of the dispersive focusing wave at some positions before the focusing point. The left plots are computed by Fourier transform and the right plots are by wavelet transform. The upper plots are in 3-D view, while the lower plots are in 2-D view.

the energy of the signal is distributed with frequency. Any local (time) information is not directly contained in Fourier transform, but is hidden in the spectrum and phase. At a certain local time, $t=t_{0}$, we have

$\eta\left(t_{0}\right)=2 \int_{0}^{\infty}|\eta(\omega)| \cos \left(\omega t_{0}+\theta(\omega)\right) \mathrm{d} \omega$.

The term inside the integral represents the amplitude spectrum and phase distribution with the frequency at a single time. Then we may define a local energy spectrum, $E\left(t_{0}, \omega\right)$, as

$E\left(t_{0}, \omega\right)=\left(|\eta(\omega)| \cos \left(\omega t_{0}+\theta(\omega)\right)\right)^{2}$,

presenting the local information of the signal directly. More generally, we will not only consider the energy at a single instant but will also analyze the energy in the neighborhood. Therefore, we will use wavelet transformation for the local energy analysis since it will show the distribution of the local energy spectrum better because it includes energy contributions from neighboring times instead of only one local time. Figures 5 and 6 illustrate the local energy distribution computed by Fourier and wavelet transform for a dispersive focusing wave and an irregular wave that will be used as study cases in Sects. 4.1 and 4.3. The plots show that the wavelet transform gives a more refined description of the local energy distribution as a function of time and frequency.
The wavelet transform is an extension of Fourier transformation. The basis function in Fourier transform is a sinusoidal of a specific frequency, and the $L^{2}$ inner product with the signal leads to the Fourier coefficient of that frequency only. A wavelet is composed of a mixture of frequencies (which is indicated by its own Fourier transform). As a consequence, the wavelet coefficients refer to this mixture of frequencies, not a single frequency. We will now provide a summary of the main notions needed in the following sections.

Definition 2.1. A mother wavelet is a zero average function, $\psi$, as

$\psi \in L^{2}(\mathbb{R}): \int \psi(t) \mathrm{d} t=0$

Definition 2.2. A wavelet family is family of functions generated from any type of mother wavelet, $\psi$, through dilatation $(s>0)$ and translation $(u \in \mathbb{R})$ :

$\psi_{u, s}(t)=\frac{1}{\sqrt{s}} \psi\left(\frac{t-u}{s}\right)$. 

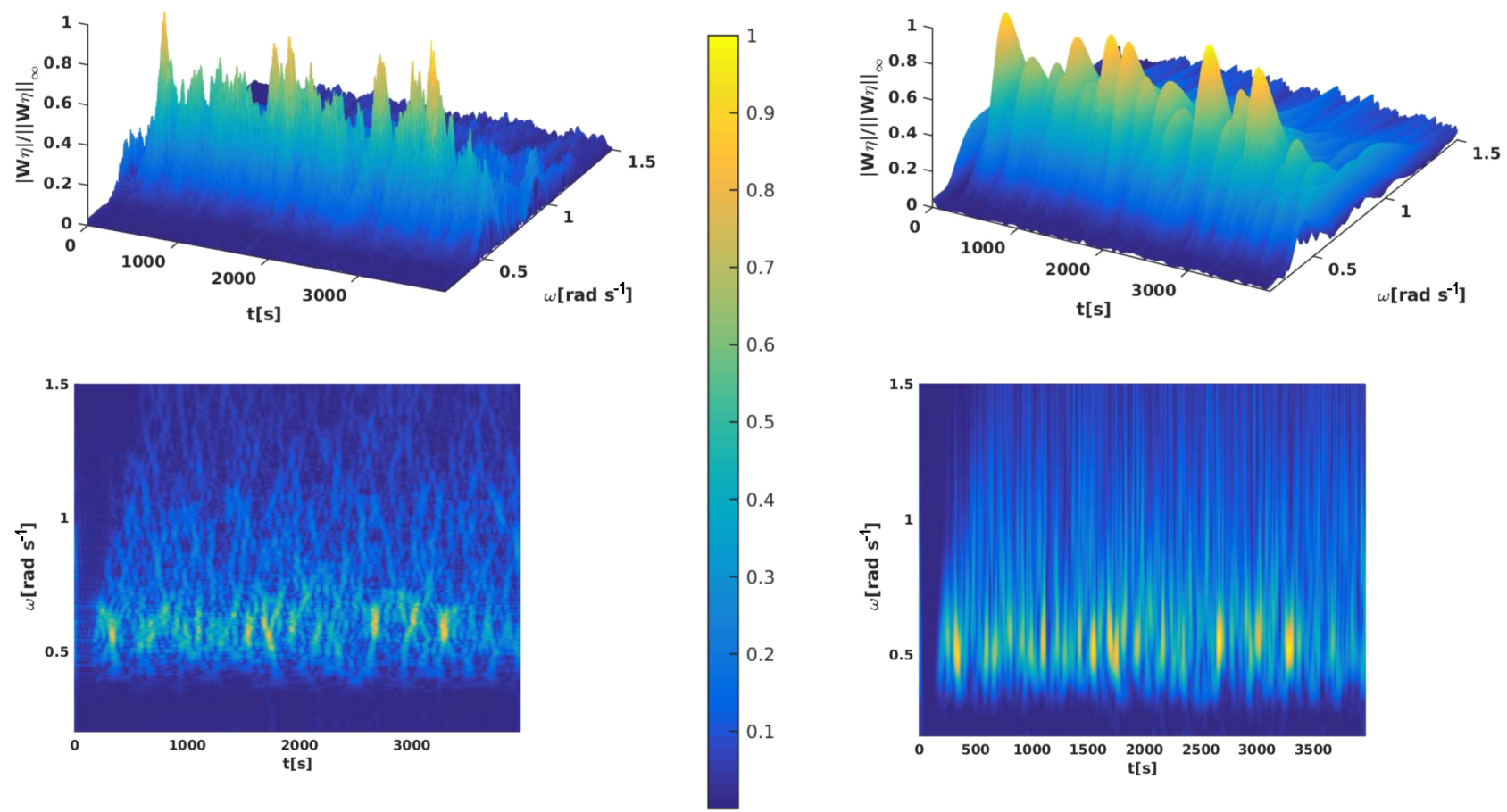

Figure 6. The same as Fig. 5; now for the irregular wave IW12 at some positions before the freak wave.

There are many types of mother wavelets: Morlet, Haar, Daubechies, Meyer, etc. (see Vialar, 2009). In this paper, we use the Morlet wavelet consisting of a plane wave modulated by a Gaussian, $\psi(t)=e^{-t^{2} / 2} e^{-i \omega_{0} t}$, which is given in the Fourier domain by $\hat{\psi}(\omega)=\sqrt{2 \pi} e^{-\left(\omega-\omega_{0}\right)^{2} / 2}$ with the central frequency $\omega_{0}$.

Definition 2.3. The continuous wavelet transform of a signal $\eta(t)$ at the scale $s$ and at the time $u$ is calculated by correlating $\eta$ with the wavelet family, $\psi_{u, s}$ :

$\mathcal{W} \eta(u, s)=\left\langle\eta, \psi_{u, s}\right\rangle=\int \eta(t) \frac{1}{\sqrt{s}} \psi^{*}\left(\frac{t-u}{s}\right) \mathrm{d} t$,

where $\psi^{*}$ is the complex conjugate of $\psi$.

From Definition 2.3, the wavelet transform of a time signal $\eta(t)$ gives a complex valued function $\mathcal{W} \eta(u, s)$. For the Morlet wavelet, we obtain

$\mathcal{W} \eta(u, s)=\int \eta(t) \frac{1}{\sqrt{s}} e^{-(t-u)^{2} / 2 s^{2}} e^{i \omega_{0}(t-u) / s} \mathrm{~d} t$.

By substituting $s=\omega_{0} / \omega$ and writing $F(t ; u, \omega)=$ $\frac{1}{\sqrt{s(\omega)}} e^{-(t-u)^{2} / 2 s(\omega)^{2}}$, the equation above gives

$\mathcal{W} \eta(u, \omega)=\int \eta(t) F(t ; u, \omega) e^{i \omega(t-u)} \mathrm{d} t$.

The function $F(t ; u, \omega)$ applies as a Gaussian window function to the signal $\eta(t)$. This shows that the wavelet transform can be interpreted as the Fourier transform of a windowed signal in the neighborhood of $t=u$. The magnitude of the wavelet transform, $|\mathcal{W} \eta(u, \omega)|$, represents the energy distribution of the signal over frequency and time and its angle, $\arg (\mathcal{W} \eta(u, \omega))$, represents the local phase of the signal.

Similar to Fourier transform, it is possible to rebuild the signal from the wavelet transform, the so-called inverse wavelet transform. It is given by

$$
\begin{aligned}
\eta(t) & =\frac{1}{C_{\psi}} \int_{0}^{\infty} \int \mathcal{W} \eta(u, s) \frac{1}{\sqrt{s}} \psi\left(\frac{t-u}{s}\right) \mathrm{d} u \frac{\mathrm{d} s}{s^{2}} \\
& =\int_{0}^{\infty}\left[\frac{1}{\omega_{0} C_{\psi}} \int \mathcal{W} \eta(u, \omega) F(t ; u, \omega) e^{-i \omega(t-u)} \mathrm{d} u\right] \mathrm{d} \omega,
\end{aligned}
$$

with

$C_{\psi}=\int_{0}^{\infty} \frac{|\hat{\psi}(\omega)|^{2}}{\omega} \mathrm{d} \omega$.

As an example, for $\omega_{0}=6$ the Morlet wavelet above produces $C_{\psi} \approx 1.883$. Different from Eq. (4) that gives the local energy spectrum computed at one time, Eq. (7) shows that the local energy spectrum from the wavelet transform is not only computed at the local time but it also includes the contribution of the signal surrounding that time.

The choice of the central frequency $\omega_{0}$ should be such that the Morlet wavelet satisfies the admissibility condition, 
$C_{\psi}<\infty$, which is equivalent to $\hat{\psi}(0)=0$. Then the (real) wavelet transform is complete and preserves the quantity of energy:

$$
\int|\eta(t)|^{2} \mathrm{~d} t \approx \frac{1}{\omega_{0} C_{\psi}} \int_{0}^{\infty} \int|\mathcal{W} \eta(u, \omega)|^{2} \mathrm{~d} u \mathrm{~d} \omega .
$$

Actually, the Morlet wavelet satisfies the condition only approximately because $\hat{\psi}(0)=\sqrt{2 \pi} e^{-\omega_{0}^{2} / 2}$ does not vanish exactly. A proper choice of $\omega_{0}$ can make the wavelet at least practically admissible and allows one to apply it widely to the signal decomposition (Lebedeva and Postnikov, 2014; Mertins, 1999). The defined Morlet wavelet is sufficiently admissible if we choose $\omega_{0}>5$ (see Mertins, 1999), hence $\omega_{0}=6$ is taken to be sufficient since then $\hat{\psi}(\omega) \leq 3.8 \times 10^{-8}$ for $\omega \leq 0$. Parseval's identity gives a relation between the signal and its Fourier transform as

$$
\begin{aligned}
\int|\eta(t)|^{2} \mathrm{~d} t & =2 \pi \int|\eta(\omega)|^{2} \mathrm{~d} \omega \\
& \approx \int\left[\frac{1}{\omega_{0} C_{\psi}} \int|\mathcal{W} \eta(u, \omega)|^{2} \mathrm{~d} u\right] \mathrm{d} \omega .
\end{aligned}
$$

Therefore, the spectral energy density of a signal can be computed through the wavelet transform, i.e,

$|\eta(\omega)|^{2} \approx \frac{1}{2 \pi \omega_{0} C_{\psi}} \int|\mathcal{W} \eta(u, \omega)|^{2} \mathrm{~d} u$.

This equation shows that the energy distribution from the wavelet transform behaves locally, and its integration over the time shift $u$ is approximately the spectral energy density obtained by Fourier transform.

\section{Characterizing freak waves}

The capability of the wavelet transform to represent a signal in time and frequency domain motivates us to investigate a freak wave locally. For a given signal, we identify group events which are parts of the time signal that may develop into propagating wave groups, i.e., that contain an amount of energy larger than a certain threshold. This threshold is determined such that the group event can build a freak wave if additional conditions are satisfied. We then determine the most energetic waves from each group event to see how the energy is distributed in both time and frequency. The most energetic waves will determine the evolution of the group event and whether its energy will converge or diverge. With these elements, we will be able to define the local coherence which will describe quantitatively the process of freak wave formation from a critical group event.

\subsection{Critical group events}

Holthuijsen (2007) defines a wave group as an uninterrupted sequence of waves with wave heights higher than an arbitrarily chosen, but usually high, threshold value. Instead of a

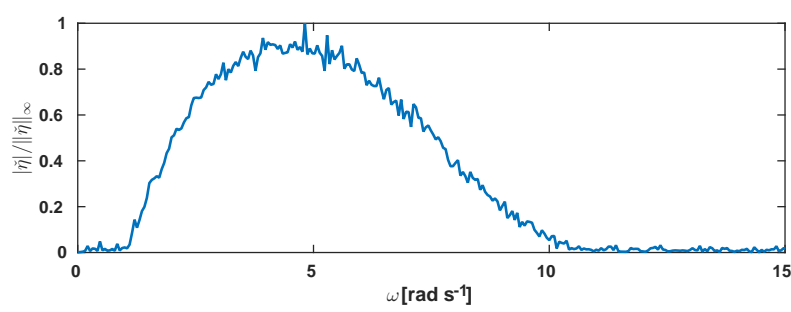

Figure 7. The normalized spectral shape of the influx signal for the case 202002.

wave group, we define a group event based on a chosen local energy level as threshold, which is determined by the contour level of the spectral energy determined by the wavelet transform. A group event of a time signal $\eta(t)$ is part of the time signal with $|\mathcal{W} \eta(u, \omega)|$ higher than a threshold value. We denote the set of group events with respect to the threshold value $\epsilon$, by $\mathrm{WG}_{\epsilon}$ :

$$
\begin{aligned}
\mathrm{WG}_{\epsilon} & =\left\{\eta_{i}(t): i=1,2, \ldots, N_{\mathrm{g}}\right\} \\
\eta_{i}(t) & =\left\{\eta(t): t=\left[t_{1}, t_{2}\right] \subset[0, T]|| \mathcal{W} \eta_{i}(u, \omega) \mid \geq \epsilon\right\} .
\end{aligned}
$$

$\mathrm{WG}_{\epsilon}$ is the assembly of $N_{\mathrm{g}}$ group events; each group is determined by the time interval during which the wavelet transform is larger than a specified value $\epsilon$. The selection of the group events depends on the chosen threshold value $\epsilon$. In practice, we normalize the value of $|\mathcal{W} \eta|$ with its maximum, so that the value of $\epsilon$ is chosen in $(0,1)$. The choice depends on the background waves since it aims to ignore the waves that do not contribute to the evolution of the group under consideration. When the background waves are high, we should choose a large $\epsilon$, but when the background waves are small, we can choose a small value of $\epsilon$. In this paper, we choose $\epsilon \approx 0.65$ for the random signals and $\epsilon \approx 0.2$ for the maximal signal.

From all the group events determined in this way, we characterize the groups that may lead to a freak wave. For a given time signal, $\eta(t), t \in[0, \mathrm{~T}]$, we define a total energy signal, $E_{\mathrm{T}}$, as

$E_{\mathrm{T}}=\int_{0}^{\mathrm{T}}|\eta(t)|^{2} \mathrm{~d} t \approx \frac{1}{\omega_{0} C_{\psi}} \int_{0}^{\mathrm{T}} \int|\mathcal{W} \eta(u, \omega)|^{2} \mathrm{~d} \omega \mathrm{d} u$.

For each value of the total energy signal, there can be a maximal wave with a coherent state.

Next, we define the total energy threshold to eliminate group events which unlikely generate a freak wave. The remaining groups are so-called critical group events.

$\mathrm{WG}_{\text {crit }}=\mathrm{WG}_{\epsilon} \cap\left\{\eta_{i}(t) \mid \int_{t_{1}}^{t_{2}} \eta_{i}(t)^{2} \mathrm{~d} t \geq \rho^{2} E_{\mathrm{T}}\right\}$

in which

$\rho=\frac{1.25 H_{\mathrm{s}}}{\int|\eta(\omega)| \mathrm{d} \omega}$ 


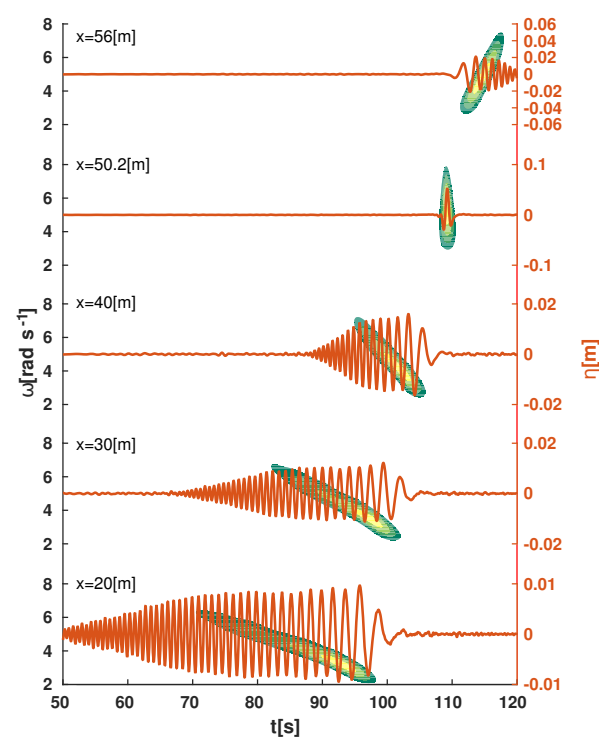

(a)

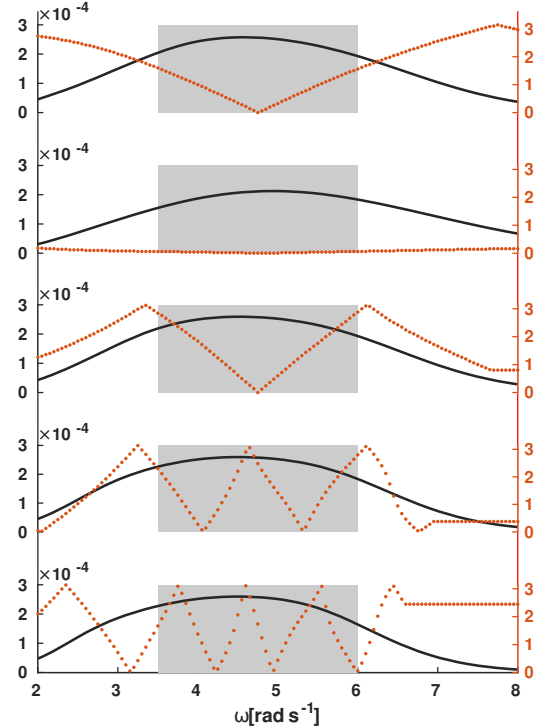

(b)

Figure 8. Case 202002. (a) Time signals at various positions of the evolution of the critical group event with the filled contour plot of wavelet spectra. The vertical axis at the left represents the wave frequency $\omega$ and the vertical axis at the right represents the surface elevation in meters. (b) The corresponding time-averaged wavelet spectra (solid line) and the time spreading (dotted line). Observe that at $x=50.2 \mathrm{~m}$ the time spreading vanishes identically in the shaded area. The shaded areas show the chosen frequency interval of the most energy carrying modes.

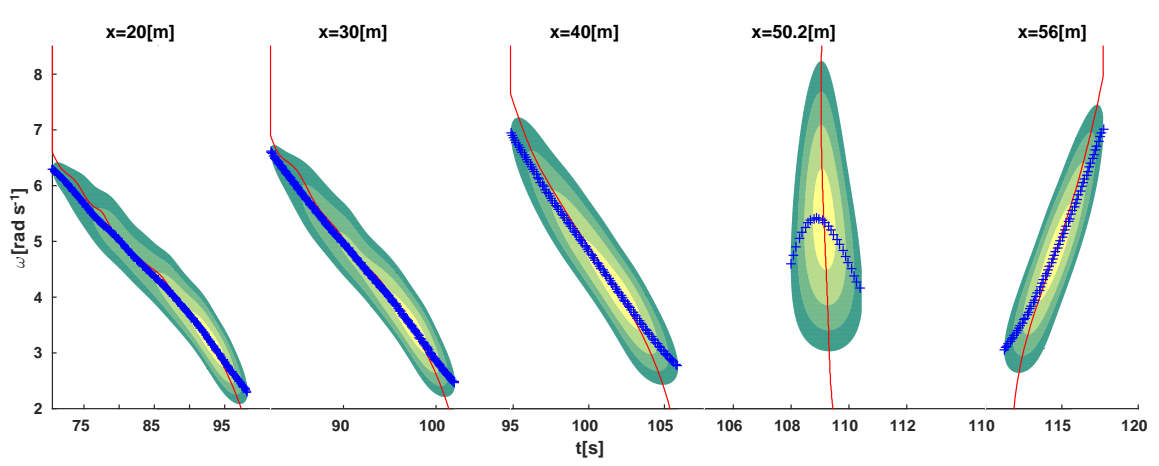

Figure 9. Case 202002. A filled contour plot of the energy distribution of the critical group event at position $x=20,30,40,50.2,56 \mathrm{~m}$. At each position, the red solid lines show the time of maximal energy at each wave frequency. The ++ lines show the wave frequency as function of time. Both are estimated by the most energetic waves in time and frequency, respectively. Before $x=50.2 \mathrm{~m}$, both solid and ++ lines show decreasing frequencies (increasing wave length) in time; then it leads to energy convergence.

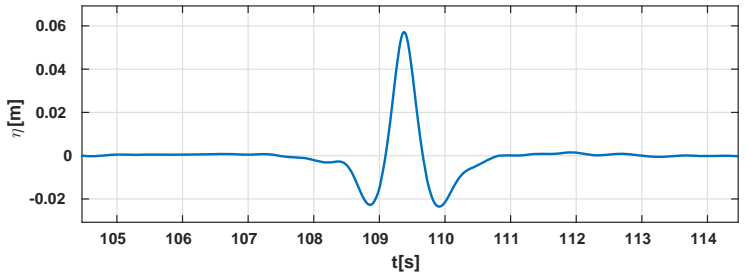

Figure 10. Case 202002. Zoomed version of the maximal wave; the crest height is $4.65 \mathrm{~m}$ and the wave height is 6.56 times the significant wave height.

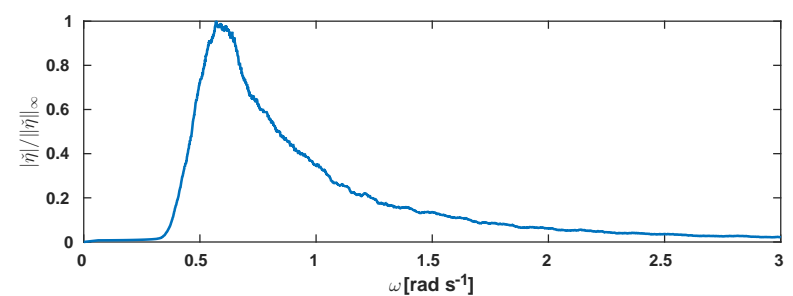

Figure 11. The same as Fig. 7; now for the case W100. 


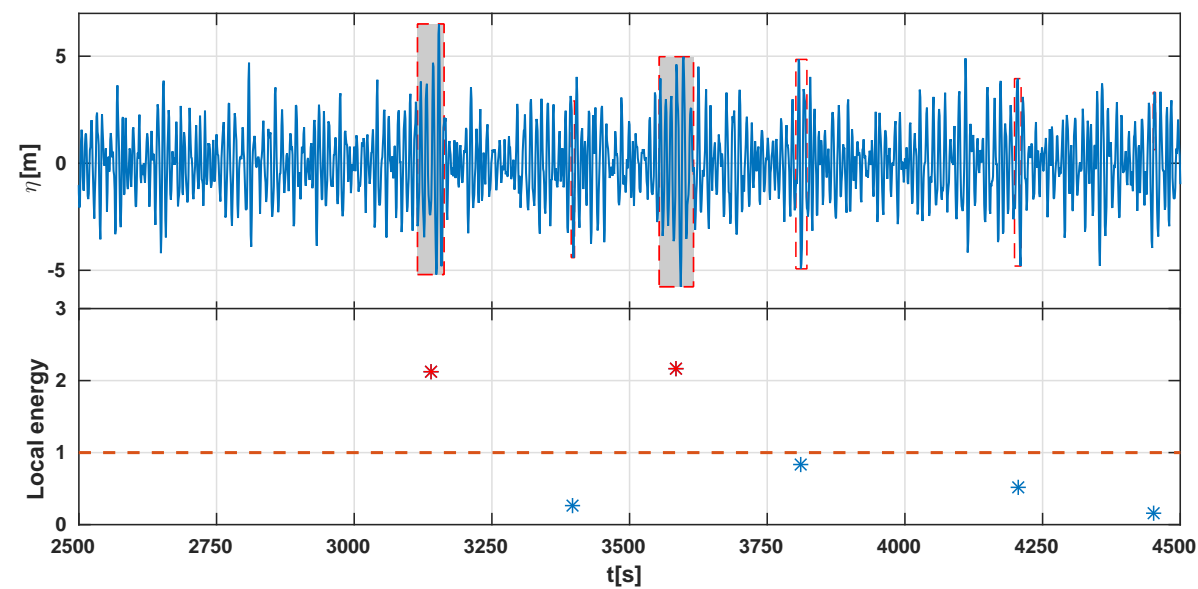

Figure 12. Case W100. Initial time signal in the interval $t \in[2500,4500] \mathrm{s}$. The critical group events are shown in the shaded areas of the upper plot. The lower plot presents the amount of local energy signal of the recognized group events compared to the local energy threshold (dashed line). The local energy signal of the critical group events is above the threshold.

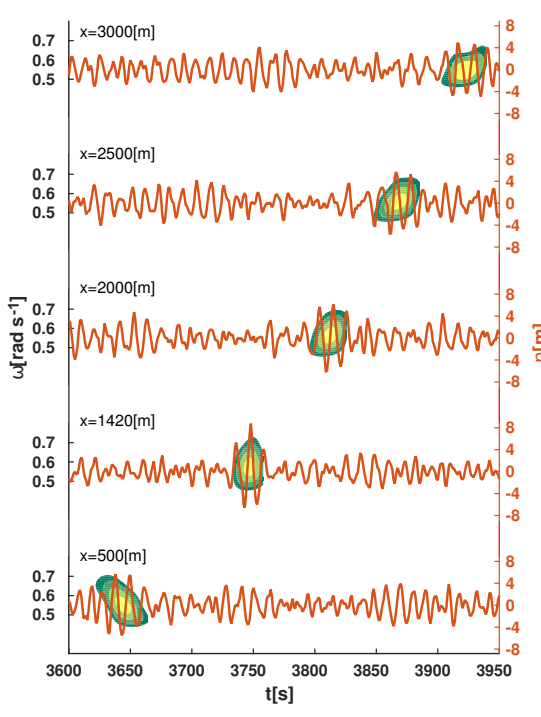

(a)

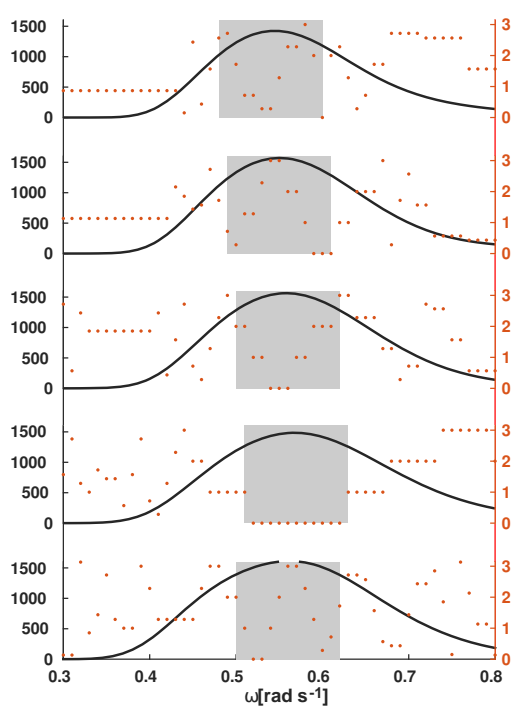

(b)

Figure 13. Case W100. (a) Time signals at various positions of the evolution of the critical group event with the filled contour plot of wavelet spectra. (b) The corresponding time-averaged wavelet spectra (solid line) and the time spreading (dotted line). Observe that at $x=1420 \mathrm{~m}$ the time spreading is zero in the shaded area. The shaded areas show the chosen frequency interval of the most energy-carrying modes.

is a freak wave threshold normalized by the amplitude of a maximal signal. Based on their local energy, these critical group events could generate a freak wave forward or backward, but the probability depends on the phases.

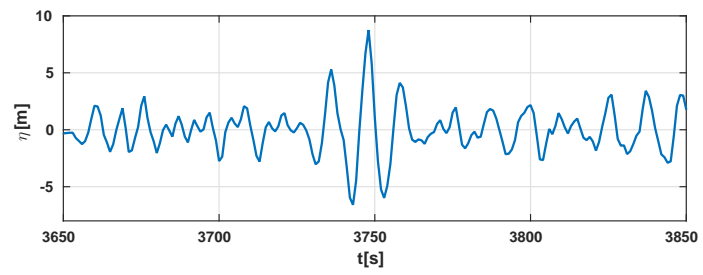

Figure 14. Case W100. Zoomed version of the freak wave; the crest height is $1.35 \mathrm{~m}$ and the wave height is 2.37 times the significant wave height. 


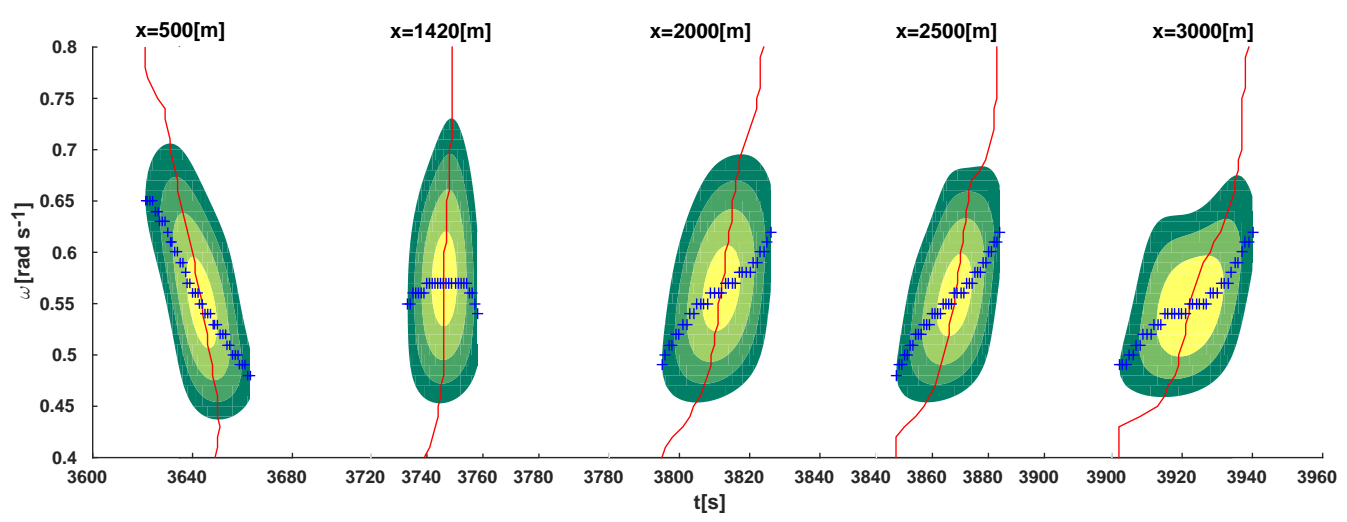

Figure 15. Case W100. A filled contour plot of the energy distribution of the critical group event at various positions. At each position, the red solid lines show the time of maximal energy at each wave frequency. The ++ lines show the wave frequency as function of time. Both are estimated by the most energetic waves in time and frequency, respectively. Both lines show a decreasing frequency before the freak wave and an increasing frequency after the freak wave, while the freak wave occurs at $x=1420 \mathrm{~m}$.

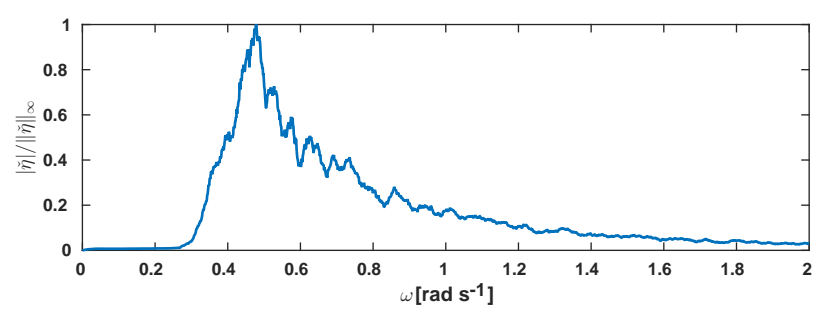

Figure 16. The same as Fig. 7; now for the case TS10000.

\subsection{Most energetic waves}

We start from the complex value of the wavelet transform of $\eta(t)$

$\mathcal{W} \eta(u, \omega)=|\mathcal{W} \eta(u, \omega)| e^{i \Theta(u, \omega)}$.

It gives the spectral energy distribution $|\mathcal{W} \eta(u, \omega)|$ and the phase information $\Theta(u, \omega)$ as a function of time and frequency. From this we may look at the frequencies that carry most energy as a function of time denoted by $\omega_{\mathrm{m}}(u)$ from the critical group event:

$\omega_{\mathrm{m}}(u)=\left\{\omega|| \mathcal{W} \eta(u, \omega)\left|=\max _{\omega}\right| \mathcal{W} \eta(u, \omega) \mid\right\}$.

Convergence of waves will occur when long waves catch up with shorter waves. Hence, when the local wave length increases, i.e., when the wave frequency decreases, the waves will converge at a later time and vice versa. Therefore, the distinction is determined by the frequency in the time interval: when decreasing in forward time, this leads to a focusing energy, and an increase leads to defocusing energy. Since continuity of the local wave frequency in the random waves cannot be guaranteed, we approximate the local wave frequency by a linear interpolation, $\omega_{\mathrm{m}}(u) \approx \widetilde{\omega}(u)$, so that we can distinguish the two cases:

$$
\begin{aligned}
& \widetilde{\omega}(u) \\
& =\widetilde{A} u+\widetilde{B}\left\{\begin{array}{ll}
\widetilde{A}>0, & \text { defocusing/diverging energy } \\
\widetilde{A} \leq 0, & \text { focusing/converging energy }
\end{array}\right. \text {. }
\end{aligned}
$$

Moreover, we can also look at the most energetic waves as a function of wave frequency. This leads to a local time of each wave contribution. In the case of a dispersive focusing wave, focusing of the energy occurs when all wave contributions are in phase at one local time.

Motivated by this, for each critical group event in a local time interval $\left[t_{1}, t_{2}\right]$, we define a function $\tau_{\mathrm{m}}(\omega)$ representing the local time of the maximal energy, $\tau_{\mathrm{m}}(\omega) \in\left[t_{1}, t_{2}\right]$, as

$\tau_{\mathrm{m}}(\omega)=\left\{u|| \mathcal{W} \eta(u, \omega)\left|=\max _{u}\right| \mathcal{W} \eta(u, \omega) \mid\right\}$.

Hence, if the critical group event gives a constant $\tau_{\mathrm{m}}(\omega)$, all frequencies contribute at the same time, which leads to local coherence at that time. If the frequencies are decreasing over the local time interval, it may indicate a local focusing at a later time.

\subsection{Local coherence}

The observations of the most energetic waves in either time or frequency can be used to see whether a freak wave may appear in forward or backward time, but the generation of a freak wave is still not assured, since the amplitude is not determined yet. The local information of the energy and phase gives a method to investigate locally the relation between the local coherence and freak wave occurrence. In this subsection, we measure the local coherence of the group event along its evolution and we will show that the highest amplitude occurs when the local coherence is maximum in the restricted frequency interval. As the wavelet transformation 


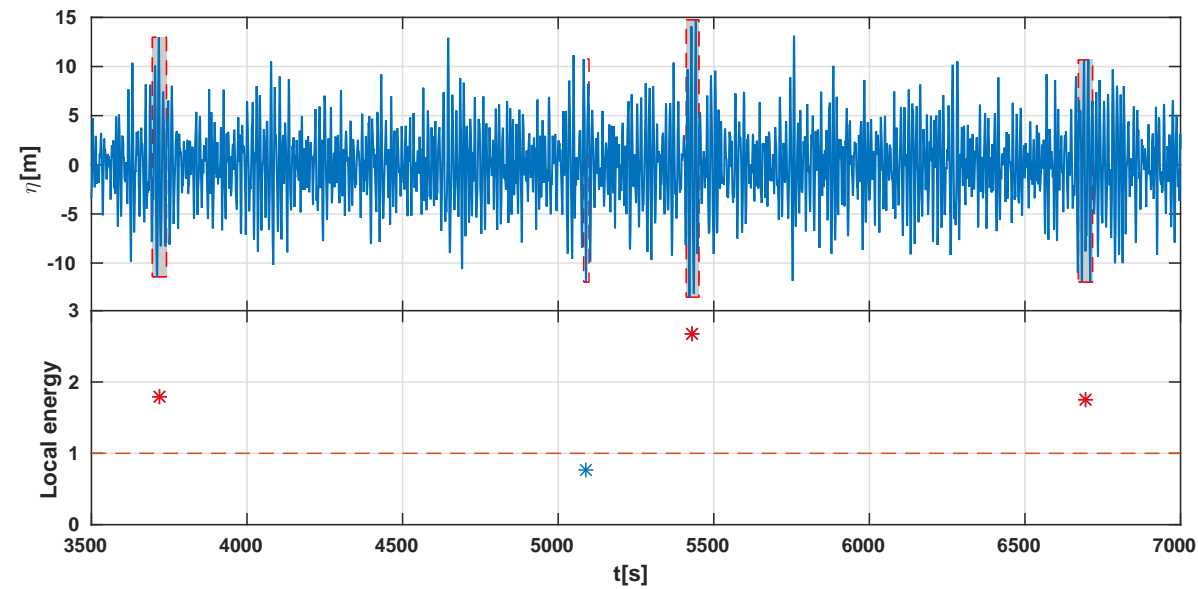

Figure 17. Case TS10000. Initial time signal in the interval $t \in[3500,7000] \mathrm{s}$. The critical group events are shown in the shaded areas of the upper plot. The lower plot presents the amount of local energy signal of the recognized group events compared to the local energy threshold (dashed line). The local energy signal of the critical group events are above the threshold.

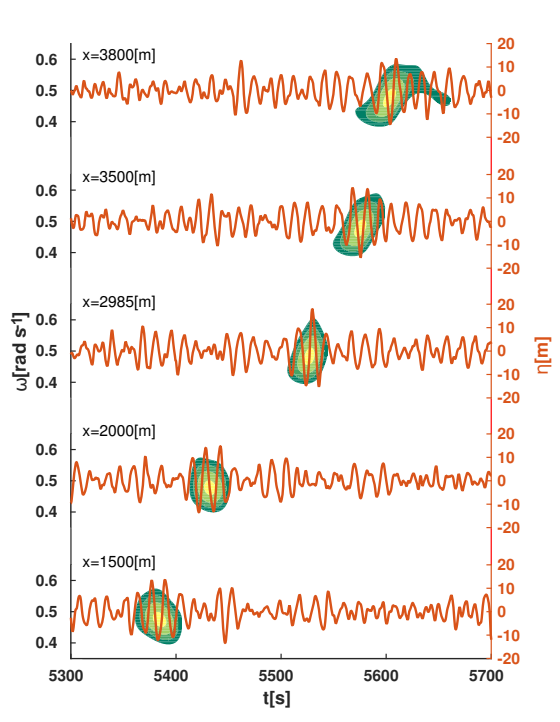

(a)

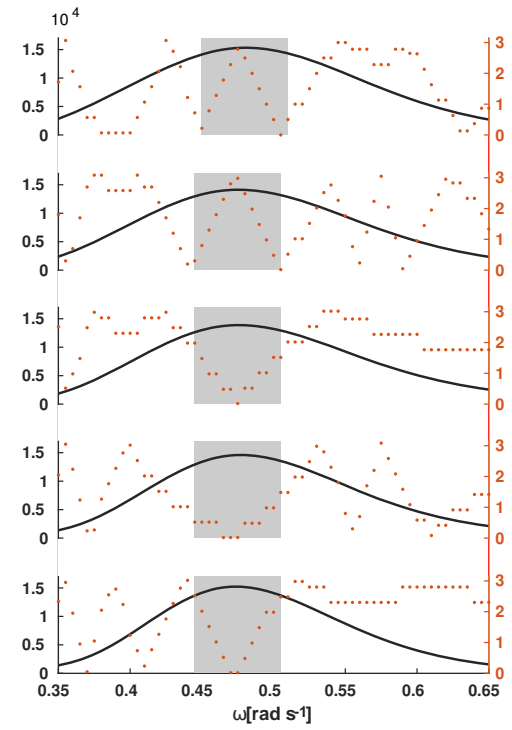

(b)

Figure 18. Case TS10000. (a) Time signals at various positions of the evolution of the critical group event with the filled contour plot of wavelet spectra. (b) The corresponding time-averaged wavelet spectra (solid line) and the time spreading (dotted line). The shaded areas show the chosen frequency interval of the most energy-carrying modes.

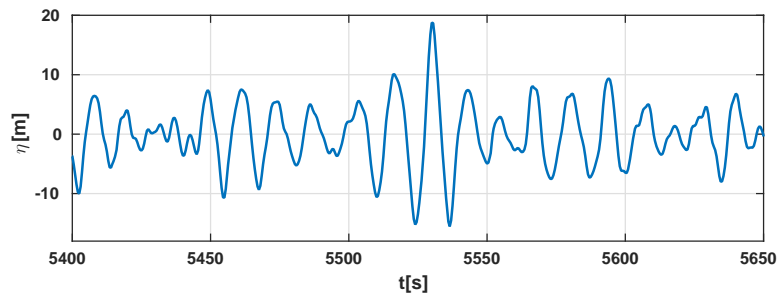

Figure 19. Case TS10000. Zoomed version of the freak wave; the crest height is $1.22 \mathrm{~m}$ and the wave height is 2.23 times the significant wave height. gives a function of frequency and time, we define a time spreading of the most energetic waves $[\varphi(\omega)]_{\tau} \in[-\pi, \pi]$ for each time $\tau \in\left(t_{1}, t_{2}\right)$ as follows:

$[\varphi(\omega)]_{\tau}=\left[\tau_{\mathrm{m}}(\omega)-\tau\right] \bmod 2 \pi$

that is taken at the time at which the absolute mean is minimal.

$\varphi(\omega)=\left\{\left.[\varphi(\omega)]_{\tau}|| \bar{\varphi}(\omega)\right|_{\tau}=\min _{\tau}|\bar{\varphi}(\omega)|_{\tau}\right\}$.

The time spreading is exactly zero at a certain frequency interval when $\tau_{\mathrm{m}}(\omega)$ is constant at that interval. To investigate 


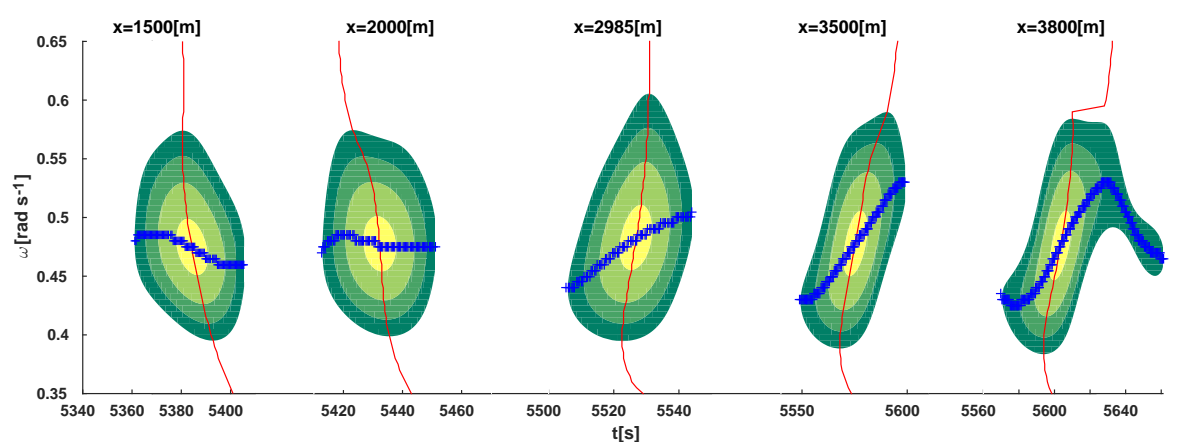

Figure 20. Case TS10000. A filled contour plot of the energy distribution of the critical group event at various positions. At each position, the red solid lines show the time of maximal energy at each wave frequency. The ++ lines show the wave frequency as function of time. Both are estimated by the most energetic waves in time and frequency, respectively.

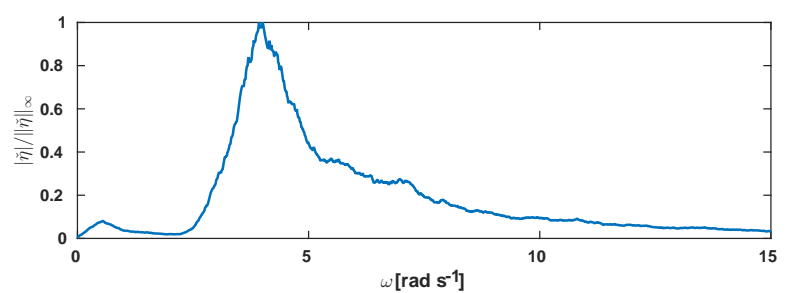

Figure 21. The same as Fig. 7; now for the case IW12.

the local coherence, we determine the maximum $(M)$, the mean $(\mu)$ and the standard deviation $(\sigma)$ of the absolute value of the time spreading normalized by $\pi$. Accordingly, we define three quantities depending on position that can represent local coherence, $\Gamma_{M, \mu, \sigma}(x) \in[0,1]$, depending on the choice for the parameters, $M, \mu$ or $\sigma$ :

$\Gamma_{M}(x)=1-M \quad \Gamma_{\mu}(x)=1-2 \mu \quad \Gamma_{\sigma}(x)=1-\sqrt{3} \sigma$.

These values represent a somewhat different measure of local coherence. Note that the extreme case $\left(\Gamma_{M}=\Gamma_{\mu}=\Gamma_{\sigma}=1\right)$ occurs for the maximal signal, when all the phases are zero. Note also that this measure is different from the degree of phase coherence defined in Latifah and van Groesen (2012), as it corresponds to the local time spreading of the most energetic waves of a group event. To investigate the dependence between the local coherence and the occurrence of freak waves, we compute the correlation between the local coherence and the maximum amplitude normalized by its timeaveraged local energy, $\operatorname{Corr}\left(\Gamma_{M, \mu, \sigma}, A_{\mathrm{m}}\right)$. For $N$ number of time signals at the positions $\left(x_{1}, x_{2}, \ldots, x_{N}\right)$, the correlation is computed by

$\operatorname{Corr}\left(\Gamma, A_{\mathrm{m}}\right)=\frac{\sum_{i=1}^{N}\left(\Gamma\left(x_{i}\right)-\mu_{\Gamma}\right)\left(A_{\mathrm{m}}\left(x_{i}\right)-\mu_{A}\right)}{(N-1) \sigma_{\Gamma} \sigma_{A}}$,

with

$A_{\mathrm{m}}\left(x_{i}\right)=\frac{\max _{t \in\left(t_{1}, t_{2}\right)}\left|\eta\left(x_{i}, t\right)\right|}{\frac{1}{t_{2}-t_{1}} \int_{t_{1}}^{t_{2}} \eta\left(x_{i}, t\right)^{2} \mathrm{~d} t}$,

and $\Gamma\left(x_{i}\right)$ is the local coherence of the time signal at $x_{i}$.

\section{Case studies}

This section presents the investigations of four study cases: an experimental dispersive focusing wave, a synthetic normal wave condition (W100), a synthetic thunderstorm condition (TS10000) and an experimental irregular wave (IW12). For each case, we start to characterize the critical group events, then we investigate the local features of these groups, namely the most energetic wave and its time spreading. We investigate the evolution of the local energy and the time spreading of each case, particulary around the critical group events, and measure the local coherence. Furthermore, we compute the correlation between the local coherence and the maximum amplitude of the group event that generates a freak wave. It will give an impression of the relevance of the parameters $\Gamma$ for measuring a freak wave.

\subsection{Focusing wave (202002)}

The case is a focusing wave that will lead to a maximal wave. We consider a dispersive focusing wave with significant wave height $0.013 \mathrm{~m}$, for which measurements at several positions are available from an experiment at MARIN (Case 202002). The experiment was executed at a water depth of $1 \mathrm{~m}$. Here, we use the elevation at the first measurement position after the wave flap as the influx signal for the numerical simulation by both the linear and nonlinear $\mathrm{AB}$ equation. The spectral shape of the influx signal with peak frequency of approximately $5 \mathrm{rads}^{-1}$ is shown in Fig. 7. The result of the numerical simulation of a dispersive focusing wave using both the linear and nonlinear $\mathrm{AB}$ equations have been previously verified with the measurements (Liam et al., 2014; Lakhturov et al., 2012).

Referring to Fig. 5, the influx signal only consists of one group event with almost zero background, which is therefore the only critical group event. This is an idealized case as the freak wave turns out to be a maximal wave that is generated from all wave components in the initial signal. This can be observed from the evolution of the influx signal; the shorter 
Table 1. Measure of the local coherence of the dispersive focusing wave.

\begin{tabular}{|c|c|c|c|c|c|c|c|c|c|c|}
\hline \multirow[t]{2}{*}{$x$} & \multicolumn{5}{|c|}{ Linear } & \multicolumn{5}{|c|}{ Nonlinear } \\
\hline & $\Gamma_{M}$ & $\Gamma_{\mu}$ & $\Gamma_{\sigma}$ & $A_{\mathrm{m}}$ & $\tilde{A}$ & $\Gamma_{M}$ & $\Gamma_{\mu}$ & $\Gamma_{\sigma}$ & $A_{\mathrm{m}}$ & $\widetilde{A}$ \\
\hline 20 & 0.009 & 0.044 & 0.504 & 0.012 & - & 0.002 & 0.009 & 0.04 & 0.49 & - \\
\hline 30 & 0.002 & 0.116 & 0.516 & 0.019 & - & 0.001 & 0.002 & 0.12 & 0.52 & - \\
\hline 40 & 0.001 & 0.231 & 0.507 & 0.046 & - & 0.001 & 0.001 & 0.23 & 0.51 & - \\
\hline 45 & 0.312 & 0.285 & 0.659 & 0.113 & - & 0.293 & 0.29 & 0.27 & 0.65 & - \\
\hline $50.05 / 50.2$ & 0.987 & 0.996 & 0.994 & 0.692 & + & 0.975 & 0.98 & 0.97 & 0.99 & + \\
\hline 56 & 0.230 & 0.208 & 0.627 & 0.097 & + & 0.281 & 0.28 & 0.24 & 0.64 & + \\
\hline $\operatorname{Corr}\left(A_{\mathrm{m}}(x), \Gamma\right)$ & 0.95 & 0.96 & 0.94 & 1 & & 0.93 & 0.94 & 0.92 & 1 & \\
\hline
\end{tabular}

(slower) waves are followed by longer (faster) waves such that at the focusing point at $50.2 \mathrm{~m}$ all waves have vanishing phase. See Fig. 8 for various plots of snapshots of the dynamics at successive measurement positions.

During the evolution, the changes of the distribution of the local energy in the time-frequency frame are described well by the filled contour plot of the local energy. The local energy distribution from one group event is squeezed into a maximal wave. This is also shown by the decreasing width of the time intervals towards the focusing point in Fig. 9. We can see at $x=20 \mathrm{~m}$ that the energy is distributed in $20 \mathrm{~s}$, at $x=40 \mathrm{~m}$ it is distributed approximately in $10 \mathrm{~s}$ and at the focusing point the energy is only distributed in $3 \mathrm{~s}$. Moreover, the pure maximal wave is shown by the zeroes of the time spreading at $x=50.2 \mathrm{~m}$ in Fig. $8 \mathrm{~b}$. The profile of the maximal wave can be seen in Fig. 10.

In order to show that the occurrence of the freak wave is related to a local coherence, and to illustrate the three different measures of coherence introduced above, we show the evolution of these coherence measures for the linear and nonlinear evolution in Table 1. It can be observed from this table that the correlation of each of the three measures of coherence and the occurrence of the maximum amplitude at $x=50.2 \mathrm{~m}$ is very strong $(\approx 0.95)$, although outside the focusing position the values of the three $\Gamma^{\prime}$ 's can be rather different. $\Gamma_{M}$ and $\Gamma_{\mu}$ seem to be much better indicators for the focusing than $\Gamma_{\sigma}$.

\subsection{Synthetic signals}

The second and third case are synthetic signals of irregular waves that are generated from a Jonswap spectrum with normal and thunderstorm sea conditions at a water depth of $480 \mathrm{~m}$ (deep water). The wave evolutions are computed linearly by the $\mathrm{AB}$ equation as the nonlinear effect for these cases is not significant. However, a freak wave is still found in both cases.

\subsubsection{Normal sea (W100)}

The initial time signal is generated from a Jonswap spectrum with time period $11.3 \mathrm{~s}, \gamma=1.9$ and significant wave height $6.3 \mathrm{~m}$ (van 't Veer and Vlasveld, 2014). The spectral shape of the initial signal is shown in Fig. 11. The duration of the time signal is approximately $3 \mathrm{~h}$. From the initial time signal, there are nine critical group events, of which the two largest groups will be investigated. We do not investigate the other critical group events since their amount of the local energy signal is slightly equal to the threshold such that they are unlikely to develop a freak wave.

Figure 12 shows the two critical groups of the influx signal with approximately the same amount of local energy signal; one is around $t=3200 \mathrm{~s}$ and the other is at $t \approx 3600 \mathrm{~s}$. Those are the most probable group events that can develop a freak wave. In the observation of the contour energy distribution, the preceding group event gives a positive $\widetilde{A}$ while the other one gives a negative value. Therefore, the critical group event around $t=3600 \mathrm{~s}$ is the candidate to generate a larger amplitude in forward time. The evolution of this critical group together with its energy distribution is shown in Fig. 13a and the changes of its time spreading are in Fig. 13b. We observe that at the freak wave position $(x=1420 \mathrm{~m})$, the time spreading is almost zero for the wave-carrying modes. Outside the freak wave position, the time spreading of the critical group event is distributed in $[0, \pi]$. The freak wave is shown in Fig. 14.

In this case, the occurrence of the freak wave can also be observed from the most energetic wave in either time or frequency (see Fig. 15). Before the freak wave, the most energetic waves give a decreasing wave frequency and after the freak wave, an increasing wave frequency occurs. At $x=1420 \mathrm{~m}$, the local time of the maximal energy is almost constant for the carrying wave modes $(\omega \in[0.5 ; 0.7])$, therefore its time spreading is nearly coherent and it generates a freak wave.

Furthermore, we investigate the change of the local coherence of the critical group event during its $3 \mathrm{~km}$ linear wave evolution. The measure of coherence at various positions is shown in Table 2 and the correlation between the local coher- 
Table 2. Measure of the local coherence of the normal sea condition wave.

\begin{tabular}{cccccc}
\hline$x$ & $\Gamma_{M}$ & $\Gamma_{\mu}$ & $\Gamma_{\sigma}$ & $A_{\mathrm{m}}$ & $\tilde{A}$ \\
\hline 500 & 0.05 & 0.10 & 0.35 & 0.01 & - \\
1420 & 0.68 & 0.90 & 0.76 & 0.018 & - \\
2000 & 0.045 & 0.167 & 0.397 & 0.010 & - \\
2500 & 0.045 & 0.175 & 0.306 & 0.008 & + \\
3000 & 0.045 & 0.028 & 0.341 & 0.007 & + \\
\hline $\operatorname{Corr}\left(A_{\mathrm{m}}(x), \Gamma\right)$ & 0.78 & 0.82 & 0.74 & 1 & \\
\hline
\end{tabular}

ence and the maximum amplitude along the evolution is presented in the lowest row. All three $\Gamma$ 's show a quite high correlation $(\geq 0.74)$ between the local coherence and the maximum amplitude. According to the correlation value, $\Gamma_{M}$ and $\Gamma_{\mu}$ seem to be better indicators for the freak wave appearance than $\Gamma_{\sigma}$.

\subsubsection{Thunderstorm sea (TS10000)}

The other synthetic signal is generated from a Jonswap spectrum with time period $13.6 \mathrm{~s}, \gamma=2$ and significant wave height $15.2 \mathrm{~m}$ (van 't Veer and Vlasveld, 2014). A snapshot of the initial time signal is shown in Fig. 17 and its spectral shape is presented in Fig. 16. This type of wave is categorized as thunderstorm sea condition, in which the appearance of a freak wave is more probable than in a normal sea condition. The duration of the initial time signal is approximately $3 \mathrm{~h}$. There are five critical group events found from the influx signal, but the two unlikely ones do not generate a freak wave since their local energy signal is not so high compared to the threshold. The largest local energy signal of the group events appears around $t \approx 5400 \mathrm{~s}$ and its maximum crest is already quite high at the initial time. Then, in forward time, it still develops to a higher crest and generates a freak wave.

Figure 18a presents the snapshots of the time signals at various positions. Also shown is the local energy distribution of the critical group event that leads to a freak wave. In Fig. 18b, the time spreading of the critical group event shows the chosen carrying wave modes $(\omega \in[0.45 ; 0.52])$. A freak wave appears at $x=2985 \mathrm{~m}$ (see Fig. 19). If we observe the time spreading at $x=2000 \mathrm{~m}$, it seems that the local time is more coherent than at the freak wave position. This can also be seen from the measure of the local coherence in Table 3 . The larger amplitude of the freak wave compared to the group event at $x=2000 \mathrm{~m}$ can be explained from its local energy distribution. The width in time of the energy spectral distribution is a bit squeezed and there is some higher wave frequency contribution which does not appear at $x=2000 \mathrm{~m}$. Figure 20 shows the filled contour plot of the local energy distribution for the most energetic waves at several positions as function of time and frequency. It can be observed that there is a change of the wave frequency order. Before the
Table 3. Measure of the local coherence of the thunderstorm condition wave.

\begin{tabular}{cccccc}
\hline$x$ & $\Gamma_{M}$ & $\Gamma_{\mu}$ & $\Gamma_{\sigma}$ & $A_{\mathrm{m}}$ & $\tilde{A}$ \\
\hline 1500 & 0.19 & 0.17 & 0.52 & 0.01 & - \\
2000 & 0.53 & 0.66 & 0.77 & 0.018 & - \\
2985 & 0.37 & 0.39 & 0.69 & 0.010 & - \\
3500 & 0.05 & 0.03 & 0.47 & 0.008 & + \\
3800 & 0.11 & 0.16 & 0.50 & 0.007 & + \\
\hline $\operatorname{Corr}\left(A_{\mathrm{m}}(x), \Gamma\right)$ & 0.80 & 0.78 & 0.81 & 1 & \\
\hline
\end{tabular}

freak wave, the short waves run ahead the long waves and after the freak wave, the short waves are behind, just as in focusing waves.

We measure the local coherences of the critical group event along its linear evolution and the results are presented in Table 3. The correlation for each local coherence $\Gamma$ and the maximum amplitude normalized by the local energy signal is quite high $(\geq 0.78)$. This shows that the appearance of the freak wave is mostly caused by the local coherence of the critical group event from the influx signal.

\subsection{Experimental signal: irregular wave (IW12)}

The fourth case is an irregular wave, for which measurements at several positions are available from MARIN experiment with a water depth of $0.6 \mathrm{~m}$ (Case 103001). It has $1.697 \mathrm{~s}$ peak period and significant wave height of approximately $0.06 \mathrm{~m}$. We use the time signal from the first measurement position after the wave flap as the influx signal. The spectral shape of the influx signal is shown in Fig. 21. The local energy distribution of the signal is presented in Fig. 6. There are six critical group events from the influx signal as shown in Fig. 22. The largest local energy signal of the wave groups is found around $t \approx 240 \mathrm{~s}$ and it develops a freak wave.

The evolution of the time signal around the critical group event and its energy distribution at several positions are shown in Fig. 23a. Even though the energy spectral distribution does not show clearly the development of the critical group event into a freak wave, the change of the time spreading shows the development of its local coherence (see Fig. 23b). A freak wave occurs at $x=103.7 \mathrm{~m}$ when its time spreading is near coherent for a short carrying wave mode. The freak wave is shown in Fig. 24. From Fig. 25, we can also see that there is unclear increasing or decreasing wave frequencies of the most energetic wave. The local coherences are measured and presented in Table 4. The three values of $\Gamma$ 's present quite high correlation $(\geq 0.75)$ between the local coherences and the maximum amplitude in both the linear and nonlinear evolution. In this case, $\Gamma_{\mu}$ performs as the best indicator for the freak wave appearance. 


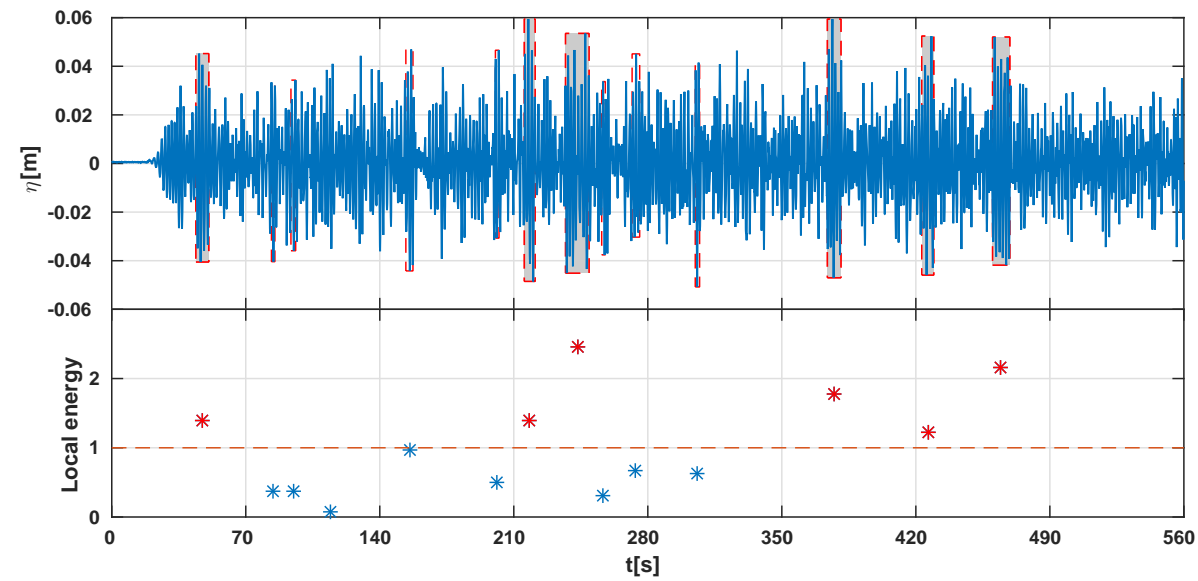

Figure 22. Case IW12. The upper plot shows the influx signal. Four critical group events are shown in the shaded areas. The lower plot shows the local energy signal of group events compared to the local energy threshold (dashed line). The local energy signal of the critical group events are above the threshold.

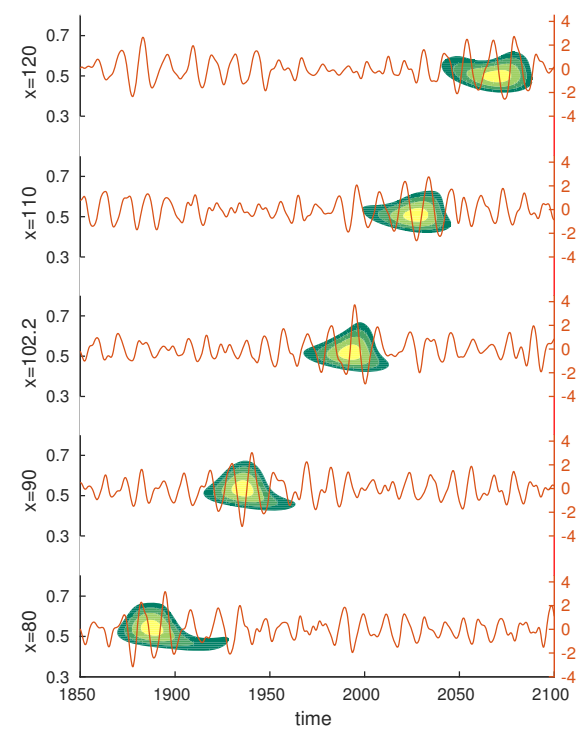

(a)

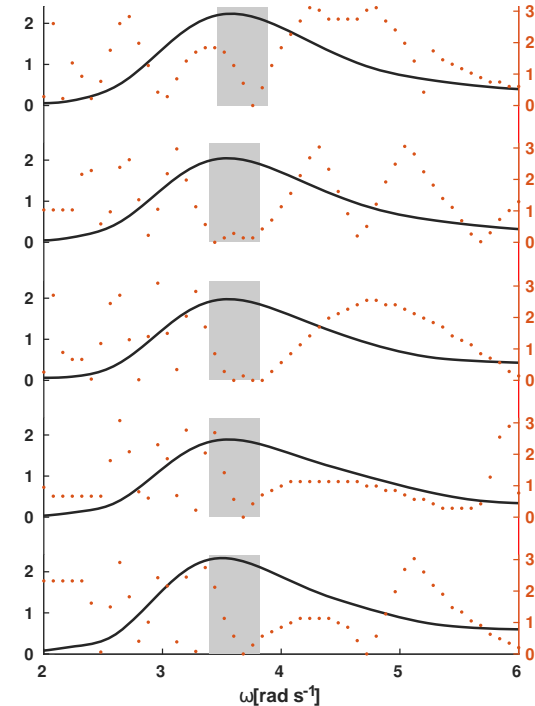

(b)

Figure 23. Case IW12. (a) Time signals at various positions of the evolution of the critical group event with the filled contour plot of wavelet spectra. (b) The corresponding time-averaged wavelet spectra (solid line) and the time spreading (dotted line). The shaded areas show the chosen frequency interval of the most energy-carrying modes.

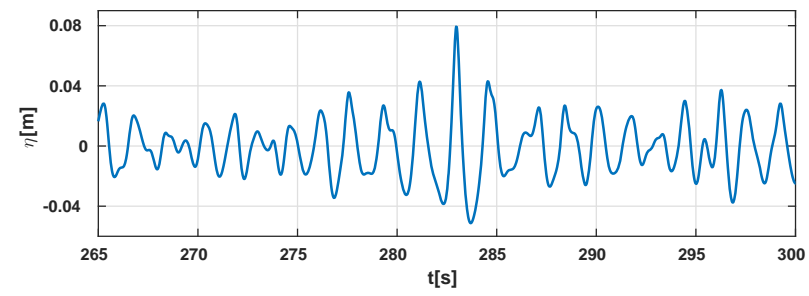

Figure 24. Case IW12. Zoomed version of the freak wave; the crest height is $1.31 \mathrm{~m}$ and the wave height is 2.15 times the significant wave height.

\section{Conclusions}

In this paper, we showed the relevance of phase coherence by illustrations of signals with increasingly less restrictions on the phase function. Then, the wavelet transform was used to determined the time-frequency spectrum of a time signal. We used the wavelet transform to identify critical group events of the influx signal and it is shown that the group event with the largest local energy signal is the most probable group to generate a freak wave. We remarked that the identification of a group event is dependent on the choice of the thresh- 
Table 4. Measure of the local coherence of IW12.

\begin{tabular}{|c|c|c|c|c|c|c|c|c|c|c|}
\hline \multirow[t]{2}{*}{$x$} & \multicolumn{5}{|c|}{ Linear } & \multicolumn{5}{|c|}{ Nonlinear } \\
\hline & $\Gamma_{M}$ & $\Gamma_{\mu}$ & $\Gamma_{\sigma}$ & $A_{\mathrm{m}}$ & $\widetilde{A}$ & $\Gamma_{M}$ & $\Gamma_{\mu}$ & $\Gamma_{\sigma}$ & $A_{\mathrm{m}}$ & $\widetilde{A}$ \\
\hline 80 & 0.19 & 0.31 & 0.46 & 0.005 & - & 0.325 & 0.511 & 0.618 & 0.005 & - \\
\hline 90 & 0.15 & 0.46 & 0.49 & 0.007 & - & 0.415 & 0.588 & 0.666 & 0.006 & - \\
\hline $102.2 / 103.7$ & 0.82 & 0.86 & 0.90 & 0.009 & - & 0.685 & 0.820 & 0.814 & 0.009 & - \\
\hline 110 & 0.55 & 0.66 & 0.74 & 0.006 & + & 0.775 & 0.833 & 0.869 & 0.006 & + \\
\hline 120 & 0.10 & 0.22 & 0.41 & 0.006 & + & 0.415 & 0.331 & 0.637 & 0.006 & + \\
\hline $\operatorname{Corr}\left(A_{\mathrm{m}}, \Gamma\right)$ & 0.78 & 0.86 & 0.78 & 1 & & 0.75 & 0.88 & 0.76 & 1 & \\
\hline
\end{tabular}

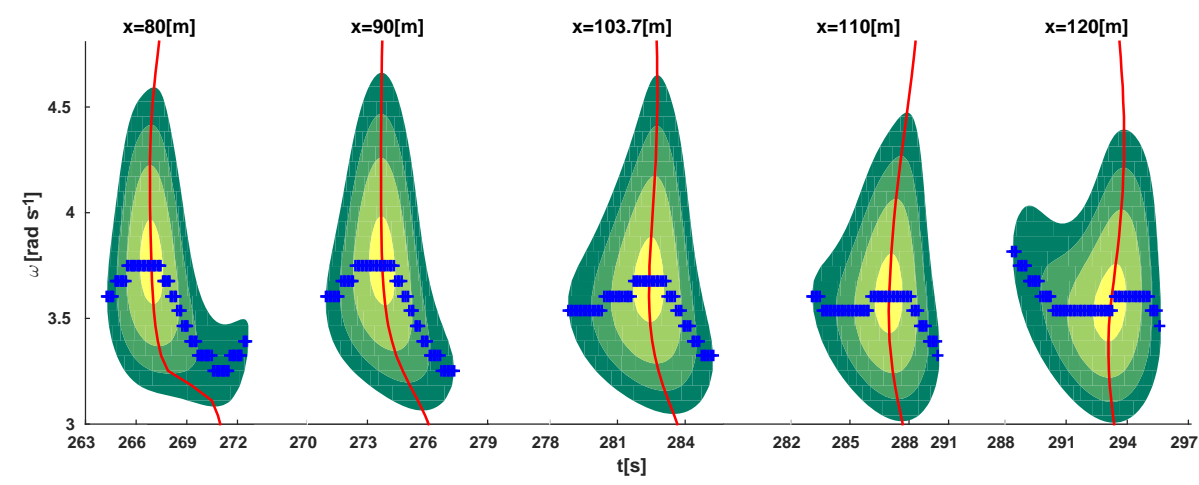

Figure 25. Case IW12. A filled contour plot of the energy distribution of the group event at various positions. At each position, the red solid lines show the time of maximal energy at each wave frequency. The ++ lines show the wave frequency as function of time. Both are estimated by the most energetic waves in time and frequency, respectively.

old value $(\epsilon)$. For irregular waves, we suggested to choose $\epsilon \approx 0.65$ and for waves with vanishing background, we could choose a smaller value $\epsilon \approx 0.2$. We defined local coherence by three parameters (the mean, maximum or standard deviation) of the time spreading of the most energetic waves from the critical group events. We investigated the change of the local coherence along its evolution and showed that all three values of the local coherence are strong indicators for the appearance of a freak wave. This indicates a local mechanism of a freak wave appearance: the freak wave is mostly developed by a local coherence of a group event. At the influx signal, the group event already contains a considerable amount of energy, which evolves into successive states with even higher coherence. Four study cases illustrate the usefulness of the introduced concepts to describe and predict the appearance of freak waves.

\section{Data availability}

The data used by this study are experimental and synthetic data. The data are freely available but not otherwise published in any publicly accessible database. The experimental data can nonetheless be provided on request by MARIN hydrodynamic laboratory, Wageningen, the Netherlands. The synthetic data can nonetheless be provided on request via email to the corresponding author Arnida L. Latifah (a.l.latifah@utwente.nl). 


\section{Appendix A: AB equation}

The $\mathrm{AB}$ equation proposed by van Groesen and Andonowati (2007) is a unidirectional wave equation above a flat bottom describing the surface wave elevation. This equation is derived by exploiting the variational formulation of surface water waves. It is accurate in second order in the wave height, applicable for finite and for infinite depth dispersion, but here we only present the equation for the finite depth. For waves above finite depth, the $\mathrm{AB}$ equation can be interpreted as a higher-order $\mathrm{KdV}$ equation; in lowest order, it is the classical $\mathrm{KdV}$ equation.

We describe the dynamics by the surface elevation, $\eta(x, t)$. The nonlinear $\mathrm{AB}$ equation can be written as

$\partial_{t} \eta= \pm \sqrt{g} A$

$\cdot\left[\eta+\frac{1}{4}(B \eta)^{2}+\frac{1}{2} B(\eta B \eta)-\frac{1}{4}(A \eta)^{2}+\frac{1}{2} A(\eta A \eta)\right]$,

where $A$ and $B$ are the pseudo-differential operators which depend on the dispersion relation; see also van Groesen et al. (2010). The linear $\mathrm{AB}$ equation is only the first term within the brackets of Eq. (A1). The minus sign in the Eq. (A1) is for the wave evolution traveling to the right and the plus sign is for the wave evolution traveling to the left. We consider dispersive wave evolution and apply the exact dispersion relation for water waves. In one space dimension, water waves on a layer of depth $h$ in a constant gravity field $g$, have dispersion given by the relation

$\omega=\Omega(k)=\operatorname{sign}(k) \sqrt{g k \tanh (k h)}$,

where $k$ is the wave number. The skew-symmetric operator $A$ and the symmetric operator $B$ are defined by

$A=C \frac{\partial_{x}}{\sqrt{g}} \quad B=\frac{\sqrt{g}}{C}$.

Here, $C$ is the phase velocity operator, i.e., the symmetric pseudo-differential operator with symbol the phase velocity $\widehat{C}$. The Fourier transform of $C$ is defined by $\widehat{C}=\frac{\Omega(k)}{k}$, therefore we can derive the Fourier transform of the operator $A$ as

$\widehat{A}=i \operatorname{sign}(k) \sqrt{k \tanh (k h)}=\frac{i \Omega(k)}{\sqrt{g}}$,

and the Fourier transform of the operator $B$ as

$\widehat{B}=\sqrt{\frac{k}{\tanh k h}}$.

The quadratic operators in the nonlinear terms of the $\mathrm{AB}$ equation cannot be easily approximated by ordinary differential operators. Thus, instead of solving the $\mathrm{AB}$ equation (A1) in physical space, the $\mathrm{AB}$ equation is solved by a pseudospectral method. 
Acknowledgements. This work was funded by the Netherlands Organisation for Scientific Research, Technology Foundation STW, number 7216. We acknowledge the MARIN hydrodynamic laboratory for their measurement data 202002 and 103001 used in this paper.

Edited by: R. Grimshaw

Reviewed by: E. Pelinovsky and one anonymous referee

\section{References}

Adcock, T. A. A., Taylor, P. H., and Draper, S.: Nonlinear dynamics of wave-groups in random seas: unexpected walls of water in the open ocean, P. R. Soc. A, 471, 20150660, doi:10.1098/rspa.2015.0660, 2015.

Bai, J., Ma, N., and Gu, X.: Evolution and Energy Transition of Focused Waves on Current, in: 34th International Conference on Ocean, Offshore and Arctic Engineering, Volume 3: Structures, Safety and Reliability, Newfoundland, Canada, 31 May-5 June 2015, Paper No. OMAE2015-41481, 8 pp., doi:10.1115/OMAE2015-41481, 2015.

Baldock, T. E., Swan, C., and Taylor, P. H.: A laboratory study of surface waves on water, Philos. T. Roy. Soc. A, 354, 649-676, 1996.

Brown, M. G. and Jensen, A.: Experiments on focusing unidirectional water waves, J. Geophys. Res., 106, 16917-16928, 2001.

Cherneva, Z. and Guedes Soares, C.: Time-frequency analysis of the sea state with the Andrea freak wave, Nat. Hazards Earth Syst. Sci., 14, 3143-3150, doi:10.5194/nhess-14-31432014, 2014.

Clauss, G. F.: Dramas of the sea: episodic waves and their impact on offshore structures, Appl. Ocean Res., 24, 147-161, 2002.

Cousins, W. and Sapsis, T. P.: Quantification and prediction of extreme events in a one-dimensional nonlinear dispersive wave model, Physica D, 280-281, 48-58, 2014.

Cousins, W. and Sapsis, T. P.: Reduced-order precursors of rare events in unidirectional nonlinear water waves, J. Fluid Mech., 790, 368-388, doi:10.1017/jfm.2016.13, 2016.

Dysthe, K., Krogstad, H. E., and Muller, P.: Oceanic rogue waves, Annual Review of Fluid Mechanics, 40, 287-310, doi:10.1146/annurev.fluid.40.111406.102203, 2008.

Garret, C. and Gemmrich, J.: Rogue waves, Phys. Today, 62, 62-63, doi:10.1063/1.3156339, 2009.

Gemmrich, J. and Garrett, C.: Unexpected Wave, J. Phys. Oceanogr., 38, 2330-2336, 2008.

Grimshaw, R., Pelinovsky, D., Pelinovsky, E., and Talipova, T.: Wave group dynamics in weakly nonlinear long-wave models, Physica D, 159, 35-57, 2001.

Grue, J., Clamond, D., Huseby, M., and Jensen, A.: Kinematics of extreme waves in deep water, Appl. Ocean Res., 25, 355-366, 2003.

Haver, S.: Freak Waves: A Suggested Definition and Possible Consequences for Marine Structures, in: Proceeding of Rogue Waves, Brest, France, 20-22 October 2004, 2004.

Holthuijsen, L. H.: Waves in Oceanic and Coastal Waters, Cambridge University Press, Cambridge, 2007.
Hu, Z., Tang, W., Xue, H., and Zhang, X.: Numerical study of Rogue waves as nonlinear Schrödinger breather solutions under finite water depth, Wave Motion, 52, 81-90, 2015.

Johannessen, T. and Swan, C.: Extreme multi-direction waves, Coastal Engineering 1998, 1110-1123, doi:10.1061/9780784404119.082, 1999.

Kharif, C. and Pelinovsky, E.: Physical mechanisms of the rogue wave phenomenon, Eur. J. Mech. B-Fluid., 22, 603-634, 2003.

Kharif, C. and Pelinovsky, E.: Waves in geophysical fluids: Tsunamis, Rogue waves, Internal waves and Internal tides, chap. Freak waves phenomenon: physical mechanisms and modelling, Springer Wien New York, 2006.

Kharif, C., Pelinovsky, E., Talipova, T., and Slunyaev, A.: Focusing of Nonlinear Wave Groups in Deep Water, JETP Letters+, 73, 170-175, 2001.

Kharif, C., Pelinovsky, E., and Slunyaev, A.: Rogue waves in the Ocean, in: Advances in Geophysical and Environmental Mechanics and Mathematics, Springer-Verlag, Berlin Heidelberg, doi:10.1007/978-3-540-88419-4, 2009.

Kwon, S., Lee, H., and Kim, C.: Wavelet transform based coherence analysis of freak wave and its impact, Ocean Eng., 32, 15721589, 2015.

Lakhturov, I., Adytia, D., and van Groesen, E.: Optimized Variational 1D Boussinesq Modelling for broad-band waves over flat bottom, Wave Motion, 49, 309-322, 2012.

Latifah, A. L. and van Groesen, E.: Coherence and predictability of extreme events in irregular waves, Nonlin. Processes Geophys., 19, 199-213, doi:10.5194/npg-19-199-2012, 2012.

Lebedeva, E. A. and Postnikov, E. B.: On alternative wavelet reconstruction formula: a case study of approximate wavelets, Royal Society Open Science, 1, 140124, doi:10.1098/rsos.140124, 2014.

Liam, L. S., Adytia, D., and van Groesen, E.: Embedded wave generation for dispersive surface wave models, Ocean Eng., 80, 7383, 2014.

Lin, E.-B. and Liu, P. C.: A discrete wavelet analysis of freak waves in the ocean, J. Appl. Math., 5, 379-394, 2004.

Liu, P. and Mori, N.: Characterizing Freak Waves with Wavelet Transform Analysis, in: Rouge Waves 2000, edited by: Olagnon, M. and Athanassoulis, G. A., Editions Ifremer: Brest, France, 2000, 151-156, 2000.

Merkoune, D., Touboul, J., Abcha, N., Mouazé, D., and Ezersky, A.: Focusing wave group on a current of finite depth, Nat. Hazards Earth Syst. Sci., 13, 2941-2949, doi:10.5194/nhess-132941-2013, 2013.

Mertins, A.: Signal Analysis: Wavelets, Filter Banks, TimeFrequency Transforms and Applications, John Wiley \& Sons Ltd, Baffins Lane, Chichester, England, 1999.

Muller, P., Osborne, A., and Garret, C.: Rogue waves, in: The 14th 'Aha Huliko'a Winter Workshop EXTREME EVENTS, 25-28 January 2005, Honolulu, Hawaii, 9 pp., 2005.

Olagnon, M. and van Iseghem, S.: Some cases of observed rogue waves and attempts to characterize their occurrence condition, in: Rogue Waves 2000: Proceedings of a Workshop in Brest, France, 29-30 November 2000, edited by: Olagnon, M. and Athanassoulis, G. A., IFREMER, 2000.

Onorato, M., Residori, S., Bortolozzo, U., Montina, A., and Arecchi, F.: Rogue waves and their generating mechanisms in different physical contexts, Phys. Rep., 528, 47-89, 2013. 
Pelinovsky, E. and Kharif, C.: Extreme Ocean Waves, Springer Netherlands, 2008.

Pelinovsky, E., Talipova, T., and Kharif, C.: Nonlinear-dispersive mechanism of the freak wave formation in shallow water, Physica D, 147, 83-94, 2000.

Pelinovsky, E., Shurgalina, E., and Chaikovskaya, N.: The scenario of a single freak wave appearance in deep water - dispersive focusing mechanism framework, Nat. Hazards Earth Syst. Sci., 11, 127-134, doi:10.5194/nhess-11-127-2011, 2011.

Porubov, A. V., Tsuji, H., Lavrenov, I. V., and Oikawa, M.: Focusing of Nonlinear Wave Groups in Deep Water, Formation of the rogue wave due to nonlinear two-dimensional waves interaction, 42, 202-210, 2005.

Prevosto, M.: Effect of Directional Spreading and Spectral Bandwidth on the Nonlinearity of the Irregular Waves, in: Proceedings of the Eighth International Offshore and Polar Engineering Conference, Montréal, Canada, 24-29 May 1998, IFREMER Brest, France, 119-123, 1998.

Ruban, V. P.: Rogue waves at low benjamin-feir indices: Numerical study of the role of nonlinearity, JETP Lett.+, 97, 686-689, 2013.

Sergeeva, A. and Slunyaev, A.: Rogue waves, rogue events and extreme wave kinematics in spatio-temporal fields of simulated sea states, Nat. Hazards Earth Syst. Sci., 13, 1759-1771, doi:10.5194/nhess-13-1759-2013, 2013.

Sergeeva, A., Slunyaev, A., Pelinovsky, E., Talipova, T., and Doong, D.-J.: Numerical modeling of rogue waves in coastal waters, Nat. Hazards Earth Syst. Sci., 14, 861-870, doi:10.5194/nhess-14861-2014, 2014.

Shemer, L., Goulitski, K., Kit, E., Grune, J., and SchmidtKoppenhagen, R.: On generation of single steep waves in tanks, in: Ocean Waves Measurement and Analysis, Fifth International Symposium Waves 2005, 3-7 July 2005, Madrid, Spain, Paper number: 113, 10 pp., 2005.
Shemer, L., Goulitski, K., and Kit, E.: Evolution of wide-spectrum unidirectional wave groups in a tank: an experimental and numerical study, Eur. J. Mech. B-Fluid., 26, 193-219, 2007.

Slunyaev, A., Pelinovsky, E., and Soares, C. G.: Modeling freak waves from the North Sea, Appl. Ocean Res., 27, 12-22, 2005.

Slunyaev, A., Didenkulova, I., and Pelinovsky, E.: Rogue Waters, Contemp. Phys., 52, 571-590, 2011.

van Groesen, E. and Andonowati: Variational derivation of $\mathrm{KdV}$ type of models for surface water waves, Phys. Let. A, 366, 195201, 2007.

van Groesen, E., Andonowati, Liam, L. S., and Lakhturov, I.: Accurate modelling of unidirectional surface waves, J. Comput. Appl. Math., 234, 1747-1756, 2010.

van 't Veer, R. and Vlasveld, E.: Green water phenomena on a TwinHull FLNG concept, in: Proceedings of the ASME 2014 33rd International Conference on Ocean, Offshore and Arctic Engineering, San Francisco, California, USA, 8-13 June 2014, Paper No. OMAE2014-23915, 9 pp., doi:10.1115/OMAE2014-23915, 2014.

Vialar, T.: Complex and Chaotic Nonlinear Dynamics, SpringerVerlag, Berlin Heidelberg, 2009.

Viotti, C., Dutykh, D., Dudley, J., and Dias, F.: Emergence of coherent wave groups in deep-water random sea, Phys. Rev. E, 87, 063001, doi:10.1103/PhysRevE.87.063001, 2013.

Wang, L., Li, J., and Li, S.: Numerical Simulation of Freak Wave Generation in Irregular Wave Train, Journal of Applied Mathematics and Physics, 3, 1044-1050, 2015.

Wu, L.-C., Lee, B.-C., Kao, C. C., Doong, D.-J., and Chang, C.C.: Applying the wavelet transform to study the features of freak waves, Coast. Eng., 32, 8 pp., 2010.

Xia, W., Ma, Y., and Dong, G.: Numerical simulation of freak waves in radom sea state, Procedia Engineering, 116, 366-372, 2015. 